\title{
DISSIPATIVE SYMMETRIZERS OF HYPERBOLIC PROBLEMS AND THEIR APPLICATIONS TO SHOCK WAVES AND CHARACTERISTIC DISCONTINUITIES*
}

\author{
YURI TRAKHININ ${ }^{\dagger}$
}

\begin{abstract}
By introducing the notations of dissipative and strictly dissipative $p$-symmetrizers of initial-boundary-value problems for linear hyperbolic systems we formalize the dissipative integrals technique [A. Blokhin, Yu. Trakhinin, in Handbook of Mathematical Fluid Dynamics, Vol. 1, North-Holland, Amsterdam, 2002, pp. 545-652] applied earlier to shock waves and characteristic discontinuities for various concrete systems of conservation laws. This enables us to prove the local in time existence of shock-front solutions of an abstract symmetric system of hyperbolic conservation laws, provided that the corresponding constant coefficients linearized problem has a strictly dissipative $p$-symmetrizer. Our result does not, in particular, require the fulfillment of Majda's block structure condition. A $p$-symmetrizer is, in some sense, a "secondary" Friedrichs symmetrizer for the symmetric system for the vector of $p$-derivatives of unknown functions, and the structure of $p$-symmetrizer takes into account (if applicable) the set of divergent constraints for the original system. After applying a $p$-symmetrizer, which is in general a set of matrices and vectors, the boundary conditions for a resulting symmetric system are dissipative (or strictly dissipative). We give concrete examples of $p$-symmetrizers. Our main examples are strictly dissipative 2-symmetrizers for shock waves in gas dynamics and magnetohydrodynamics. A general procedure for constructing a $p$-symmetrizer does not however exist. But, if it was somehow constructed, then we do not need to test the Lopatinski condition that is often connected with insuperable technical difficulties. As an illustration, we refer to the author's recent result [Yu. Trakhinin, Arch. Ration. Mech. Anal., 177 (2005), pp. 331-366] for compressible current-vortex sheets for which the construction of a dissipative 0 -symmetrizer has first enabled the finding of sufficient conditions for their weak linearized stability.
\end{abstract}

Key words. symmetric hyperbolic systems, dissipative boundary conditions, multidimensional conservation laws, shock waves

AMS subject classifications. 35L50, 35L65, 35L67

DOI. $10.1137 / 050628003$

1. Introduction: Initial-boundary-value problems for quasi-linear hyperbolic systems. Consider a system of $N$ conservation laws

$$
\partial_{t} \mathcal{P}^{0}(\mathbf{U})+\sum_{j=1}^{n} \partial_{j} \mathcal{P}^{j}(\mathbf{U})=0
$$

where $\mathcal{P}^{\alpha}=\mathcal{P}^{\alpha}(\mathbf{U})=\left(\mathcal{P}_{1}^{\alpha}, \ldots, \mathcal{P}_{N}^{\alpha}\right), \mathbf{U}=\mathbf{U}(t, \mathbf{x})=\left(u_{1}, \ldots, u_{N}\right), \mathbf{x}=\left(x_{1}, \ldots, x_{n}\right)$ $\in \mathbb{R}^{n}, \partial_{t}:=\partial / \partial t, \partial_{j}:=\partial / \partial x_{j}$. With the notation

$$
\operatorname{div} \mathbf{a}:=\sum_{j=1}^{n} \partial_{j} a^{j} \quad\left(\mathbf{a}=\mathbf{a}(t, \mathbf{x})=\left(a^{1}, \ldots, a^{n}\right) \quad \text { is a vector }\right)
$$

system (1.1) in componentwise form reads

$$
\partial_{t} \mathcal{P}_{i}^{0}(\mathbf{U})+\operatorname{div} \mathcal{P}_{i}(\mathbf{U})=0, \quad i=\overline{1, N},
$$

${ }^{*}$ Received by the editors March 29, 2005; accepted for publication (in revised form) October 25, 2005; published electronically March 24, 2006. This work was supported by EPSRC research grant GR/S96609/01.

http://www.siam.org/journals/sima/37-6/62800.html

†Sobolev Institute of Mathematics, Koptyuga pr.4, 630090 Novosibirsk, Russia (trakhin@math. nsc.ru). 
where $\mathcal{P}_{i}=\mathcal{P}_{i}(\mathbf{U})=\left(\mathcal{P}_{i}^{1}, \ldots, \mathcal{P}_{i}^{n}\right)$. Assuming that the flux functions $\mathcal{P}_{i}^{\alpha}$ are smooth enough (in practice, they are usually $C^{\infty}$ ), one can rewrite (1.1) as the quasi-linear system

$$
B_{0}(\mathbf{U}) \mathbf{U}_{t}+\sum_{j=1}^{n} B_{j}(\mathbf{U}) \mathbf{U}_{x_{j}}=0
$$

with $B_{\alpha}=\left(\partial \mathcal{P}^{\alpha} / \partial \mathbf{U}\right)$.

We will assume that system (1.1) may be supplemented (but not necessarily) by a set of $K$ divergent constraints

$$
\operatorname{div} \boldsymbol{\Psi}_{j}(\mathbf{U})=0, \quad j=\overline{1, K},
$$

where $\boldsymbol{\Psi}_{j}=\boldsymbol{\Psi}_{j}(\mathbf{U})=\left(\Psi_{j}^{1}, \ldots, \Psi_{j}^{n}\right)$. For example, for the system of gas dynamics one has no divergent constraints at all, whereas the system of magnetohydrodynamics (MHD) is supplemented by the sole $(K=1)$ divergent constraint $\operatorname{div} \mathbf{H}=0$ (see, e.g., [28]). We also refer, for instance, to Landau's equations of superfluid [29] (see also [11]) which are supplemented by the tree divergent constrains $\nabla \times \mathbf{v}_{\mathbf{s}}=0\left(\mathbf{v}_{\mathbf{s}}\right.$ is the superfluid velocity $[29,11])$.

ASSUMPTION 1.1. The divergent constraints (1.3) are the restrictions on the initial data for system (1.1). That is, if (1.3) are satisfied initially, they hold for all $t>0$.

Remark 1.1. Assumption 1.1 is quite natural because it holds for all the physically relevant models: MHD, Landau's equations of superfluid, etc. (see, e.g., [11] for further examples). In practice, (1.3) $\left.\right|_{t>0}$ is proved by applying the operator div to appropriate subsystems of (1.1) and taking into account (1.3) $\left.\right|_{t=0}$. Of course, applying div requires $n \leq N$. The last assumption will be also made for other reasons (see section 3).

Sometimes, by an appropriate choice of the vector of unknowns $\mathbf{U}$ a concrete system of conservation laws can be written in the nonconservative form (1.2) with symmetric matrices $B_{\alpha}$. For example, the systems of gas dynamics and MHD are written as symmetric systems for the vectors of unknowns $\mathbf{U}=(p, \mathbf{v}, S)$ and $\mathbf{U}=(p, \mathbf{v}, \mathbf{H}, S)$, respectively. At the same time, it is not always possible to guess an appropriate vector $\mathbf{U}$ for which a system of conservation laws, (1.1), can be rewritten as a symmetric quasi-linear system. But, as was first shown by Godunov [23, 24], system (1.1) can be always symmetrized if we know, a priori, an additional conservation law ("entropy" conservation)

$$
\partial_{t} \Phi^{0}(\mathbf{U})+\operatorname{div} \boldsymbol{\Phi}(\mathbf{U})=0
$$

with $\mathbf{\Phi}=\boldsymbol{\Phi}(\mathbf{U})=\left(\Phi^{1}, \ldots, \Phi^{n}\right)$, which holds on smooth solutions of (1.1). That is, one can find an invertible change of unknowns $\mathbf{U} \rightarrow \mathbf{Q}$ such that the system

$$
A^{0}(\mathbf{Q}) \mathbf{Q}_{t}+\sum_{j=1}^{n} A^{j}(\mathbf{Q}) \mathbf{Q}_{x_{j}}=0
$$

is symmetric: $A^{\alpha}=\left(A^{\alpha}\right)^{*}$, where

$$
A^{\alpha}=\left(\frac{\partial \mathcal{P}^{\alpha}}{\partial \mathbf{Q}}\right)=B_{\alpha}(\mathbf{U}(\mathbf{Q}))\left(\frac{\partial \mathbf{U}}{\partial \mathbf{Q}}\right),
$$


i.e., $A^{\alpha}=B_{\alpha} J^{-1}$, with $J=J(\mathbf{Q})=(\partial \mathbf{Q} / \partial \mathbf{U})$. It should be noted that if system (1.1) is accompanied by a set of divergent constraints (1.3), then these constrains should be generically taken into account under Godunov's symmetrization. For the process of symmetrization itself we refer to [13] and references therein (note that, in particular, $\left.\mathbf{Q}=\left(\partial \Phi^{0} / \partial \mathcal{P}^{0}\right)\right)$.

It is worth noting that the symmetric system (1.4) can be rewritten as a quasilinear system for the original vector of unknowns $\mathbf{U}$ that is again symmetric. Indeed, (1.4) clearly implies the system

$$
A_{0}(\mathbf{U}) \mathbf{U}_{t}+\sum_{j=1}^{n} A_{j}(\mathbf{U}) \mathbf{U}_{x_{j}}=0
$$

where the matrices $A_{\alpha}=A_{\alpha}(\mathbf{U}):=J^{*} A^{\alpha} J=J^{*} B_{\alpha}$ are symmetric. Thus, the matrix

$$
S=S(\mathbf{U})=J^{*}=\left(\frac{\partial \mathbf{Q}}{\partial \mathbf{U}}\right)^{*}
$$

is the one (called Friedrichs symmetrizer) that symmetrizes system (1.2):

$$
A_{\alpha}=S B_{\alpha}=A_{\alpha}^{*} .
$$

Recall that the quasi-linear symmetric system (1.5) is symmetric hyperbolic in the sense of Friedrichs [22] if $A_{0}(\mathbf{U})>0$ (or $A_{0}(\mathbf{U})<0$ if we multiply (1.5) by -1 ).

The main requirement for the local in time well-posedness of the Cauchy problem for a quasi-linear system of conservation laws is the hyperbolicity condition that is easily verified for symmetric systems. The local existence theorem for the Cauchy problem for symmetric hyperbolic systems was independently proved by Vol'pert and Khudyaev [47], Lax [30], and Kato [26] (see also [34]). In contrast with the Cauchy problem, the conditions for well-posedness of initial-boundary-value problems for hyperbolic systems, in the generic case, cannot be easily found even for linearized problems with constant coefficients.

1.1. Standard boundary conditions. Let us first consider quasi-linear initialboundary-value problems with standard boundary conditions [40]:

$$
\begin{gathered}
L(\mathbf{U}) \mathbf{U}=0 \quad \text { in }[0, T] \times \mathbb{R}_{+}^{n}, \\
M\left(t, \mathbf{x}^{\prime}, \mathbf{U}\right) \mathbf{U}=0 \quad \text { on }[0, T] \times\left\{x_{1}=0\right\} \times \mathbb{R}^{n-1}, \\
\left.\mathbf{U}\right|_{t=0}=\mathbf{U}_{0} \quad \text { in } \mathbb{R}_{+}^{n},
\end{gathered}
$$

where $L=L(\mathbf{U})=A_{0}(\mathbf{U}) \partial_{t}+\sum_{j=1}^{n} A_{j}(\mathbf{U}) \partial_{j}$, and system (1.6a) is supposed to be symmetric hyperbolic, and the matrix $M$ is a $d \times N$ matrix. Here and below $\mathbb{R}_{ \pm}^{n}=\left\{x_{1} \gtrless 0, \mathbf{x}^{\prime} \in \mathbb{R}^{n-1}\right\}, \mathbf{x}^{\prime}=\left(x_{2}, \ldots, x_{n}\right)$. Without loss of generality we consider the problem in a half-space because the case of a smooth bounded domain $\Omega$ is reduced, in some sense, to problem (1.6) by a finite partition of unity subordinated to an open covering of $\bar{\Omega}$.

To prove a local (in time) existence theorem for problem (1.6) we should consider the following linear problem associated to (1.6):

$$
\begin{gathered}
L(\widehat{\mathbf{U}}) \mathbf{U}=\mathbf{f} \quad \text { in }[0, T] \times \mathbb{R}_{+}^{n}, \\
M\left(t, \mathbf{x}^{\prime}, \widehat{\mathbf{U}}\right) \mathbf{U}=\mathbf{g} \quad \text { on }[0, T] \times\left\{x_{1}=0\right\} \times \mathbb{R}^{n-1}, \\
\left.\mathbf{U}\right|_{t=0}=\mathbf{U}_{0} \quad \text { in } \mathbb{R}_{+}^{n},
\end{gathered}
$$

where $\widehat{\mathbf{U}}$ is a given vector-function. Here we introduce the source terms $\mathbf{f}(t, \mathbf{x})$ and 
$\mathbf{g}\left(t, \mathbf{x}^{\prime}\right)$ to make the interior equations and the boundary conditions inhomogeneous (this is needed to attack the nonlinear problem).

Recall that the boundary conditions (1.7b) are called dissipative if

$$
-\left.\left(A_{1} \mathbf{U}, \mathbf{U}\right)\right|_{x_{1}=0} \geq 0 \quad \forall \mathbf{U} \in \operatorname{ker} M,
$$

where $-A_{1}=-A_{1}(\widehat{\mathbf{U}})$ is the boundary matrix, $M=M\left(t, \mathbf{x}^{\prime}, \widehat{\mathbf{U}}\right)$. They are strictly dissipative if there exist a fixed constant $\gamma>0$ such that

$$
-\left.\left(A_{1} \mathbf{U}, \mathbf{U}\right)\right|_{x_{1}=0} \geq \gamma|\mathbf{V}|^{2} \quad \forall \mathbf{U} \in \operatorname{ker} M,
$$

where $\mathbf{V}$ is the "noncharacteristic part" of the trace $\left.\mathbf{U}\right|_{x_{1}=0}$, i.e., the projection of $\left.\mathbf{U}\right|_{x_{1}=0}$ orthogonal to ker $\left.A_{1}\right|_{x_{1}=0}(\mathbf{V}=\mathbf{U}$ for the case of noncharacteristic boundary, i.e., when $\left.\operatorname{det} A_{1}\right|_{x_{1}=0} \neq 0$ ). Recall also that the boundary conditions (1.7b) are called maximally dissipative if they are dissipative and

$$
\operatorname{dim} \operatorname{ker} M=\# \text { nonpositive eigenvalues of }\left.A_{1}\right|_{x_{1}=0} \text { counting multiplicity }
$$

(we use the definition from [39]). Property (1.10) means that the hyperbolic system (1.7a) has the correct number of boundary conditions in (1.7b), i.e.,

$$
d=\# \text { positive eigenvalues of }\left.A_{1}\right|_{x_{1}=0} \text { counting multiplicity. }
$$

In the following we always assume that the number of boundary conditions is correct and we therefore drop the word "maximally" when speaking about dissipative or strictly dissipative boundary conditions.

For the inhomogenous boundary conditions (1.7b) inequality (1.9) implies that there exists a fixed constant $\delta>0$ such that

$$
-\left.\left(A_{1} \mathbf{U}, \mathbf{U}\right)\right|_{x_{1}=0} \geq \delta|\mathbf{V}|^{2}-\delta^{-1}|\mathbf{g}|^{2}
$$

for all $\mathbf{U}$ satisfying (1.7b). To deduce inequality (1.11) from (1.9) it needs to reduce system (1.7a) to the form [35], $\mathcal{A}_{0} \mathbf{W}_{t}+\sum_{j=1}^{n} \mathcal{A}_{j} \mathbf{W}_{x_{j}}=\ldots$, where $\mathbf{U}=\mathcal{T} \mathbf{W}, \mathcal{A}_{\alpha}=$ $\mathcal{T}^{*} A_{\alpha} \mathcal{T}, \mathcal{A}_{1}=\operatorname{diag}\left(D_{1},-D_{2}, 0\right), D_{i}>0,\left(\mathcal{A}_{1} \mathbf{W}, \mathbf{W}\right)=\left(D_{1} \mathbf{W}_{1}, \mathbf{W}_{1}\right)-\left(D_{2} \mathbf{W}_{2}, \mathbf{W}_{2}\right)$, and the boundary conditions $(1.7 \mathrm{~b})$ are supposed to be rewritten in the form $\mathbf{W}_{1}=$ $S \mathbf{W}_{2}+\tilde{\mathbf{g}}$. Analogous simple arguments show that the dissipativity hypothesis (1.8) implies that there is a matrix $B=B\left(t, \mathbf{x}^{\prime}\right)$ such that

$$
-\left.\left(A_{1} \mathbf{U}, \mathbf{U}\right)\right|_{x_{1}=0} \geq(B \mathbf{g}, \mathbf{V})-\gamma|\mathbf{g}|^{2}
$$

for all $\mathbf{U}$ satisfying (1.7b), where $\gamma$ is a constant, the matrix $B$ can be, in principle, explicitly written out (it depends on $\mathcal{T}$, etc.).

If the boundary conditions (1.6b) are linear, i.e., $M=M\left(t, \mathbf{x}^{\prime}\right)$, then the boundary conditions for the associated linear problem (1.7) can be considered to be homogenous $(\mathbf{g}=0)$ and the dissipativity hypothesis (1.8) is quite enough to prove a local existence theorem for problem (1.6) by standard fixed-point argument. In this case, for the linear problem (1.7) the basic estimate following from assumption (1.8) reads [40]

$$
\|\mathbf{U}(t)\|_{L_{2}\left(\mathbb{R}_{+}^{n}\right)} \leq C\left\{\left\|\mathbf{U}_{0}\right\|_{L_{2}\left(\mathbb{R}_{+}^{n}\right)}+\|\mathbf{f}\|_{L_{2}\left([0, T] \times \mathbb{R}_{+}^{n}\right)}\right\},
$$

where $C=C(T)$ is a positive constant independent of the initial data and the source terms. For linear boundary conditions on a noncharacteristic boundary the local $W_{2}^{s}$ existence theorem for problem (1.6) was proved by Schochet [41] (see Appendix A 
of [41]), where $s \geq[n / 2]+2$ as for the Cauchy problem [47,30,26]. The case of characteristic boundary was considered by Secchi [42] (he has proved a local existence theorem in anisotropic weighted Sobolev spaces [42]).

However, if the boundary conditions in (1.6b) are nonlinear, i.e., $M$ depends on $\mathbf{U}$, then one has to consider inhomogenous boundary conditions in the linear problem (1.7) (even if the original conditions (1.6b) were homogenous). The usual way (see, e.g., [40]) to deal with inhomogenous boundary conditions suggests to subtract from the solution a more regular function satisfying the boundary conditions, and reduce problem (1.7) to one with homogenous boundary conditions. But, such a way leads to the loss of " $1 / 2$ derivative" from $\mathbf{g}$ (see [40]). That is, the dissipativity hypothesis is already not enough to achieve a nonlinear local existence result by standard iterations.

At the same time, when the boundary conditions in (1.6b) are nonlinear, but conditions (1.7b) are strictly dissipative, using inequality (1.11), we can easily deduce the basic a priori estimate (with no loss of derivatives)

$$
\|\mathbf{U}(t)\|_{L_{2}\left(\mathbb{R}_{+}^{n}\right)} \leq C\left\{\left\|\mathbf{U}_{0}\right\|_{L_{2}\left(\mathbb{R}_{+}^{n}\right)}+\|\mathbf{f}\|_{L_{2}\left([0, T] \times \mathbb{R}_{+}^{n}\right)}+\|\mathbf{g}\|_{L_{2}\left([0, T] \times \mathbb{R}^{n-1}\right)}\right\}
$$

and its higher order counterparts (see Appendix A in [43]). This enables one to prove a local existence theorem for problem (1.6) by standard fixed-point argument. The proof for the general problem (1.6) has not been written out somewhere, but we can refer to [43] for a concrete example of problem (1.6). Note that the well-posedness of the linear problem (1.7) with $\mathbf{g} \neq 0$ was proved in Appendix A of [43].

That is, when conditions (1.7b) are inhomogenous but strictly dissipative, an unpleasant loss of derivatives is avoided by a direct approach to the original problem (1.7) with inhomogenous boundary conditions (for problem (1.7) this was done in Appendix A of [43]; see also section 4 for shock waves). If, however, conditions (1.7b) are just dissipative (but not strictly dissipative), such a direct approach to the linear problem (1.7) only enables us to obtain the a priori estimate with the loss of one derivative from $\mathbf{g}$ :

$$
\|\mathbf{U}(t)\|_{W_{2}^{1}\left(\mathbb{R}_{+}^{n}\right)} \leq C\left\{\left\|\mathbf{U}_{0}\right\|_{W_{2}^{1}\left(\mathbb{R}_{+}^{n}\right)}+\|\mathbf{f}\|_{W_{2}^{1}\left([0, T] \times \mathbb{R}_{+}^{n}\right)}+\|\mathbf{g}\|_{W_{2}^{2}\left([0, T] \times \mathbb{R}^{n-1}\right)}\right\} .
$$

Here we suppose that the boundary is noncharacteristic. For the case of characteristic boundary the counterpart of (1.14) indicates a loss of control on derivatives in the normal direction $[39,42,16]$. To deduce estimate (1.14) we should differentiate problem (1.7) with respect to $t$ and $\mathbf{x}^{\prime}$. Then, we take into account inequality (1.12) and integrate by parts the boundary integral (see section 3 for the case of Rankine-Hugoniot boundary conditions).

Remark 1.2. For the case of linearized Rankine-Hugoniot boundary conditions a priori estimates with loss of derivatives were proved for shock waves and characteristic discontinuities by Coulombel [17], Coulombel and Secchi [19], and the author [45] (in [45] the a priori estimates are formally with no loss of derivatives because the boundary conditions were supposed to be homogenous). For the case of standard boundary conditions, cf. (1.7b), the a priori estimate (1.14) is a basic estimate, and it is not difficult to obtain an estimate for higher order derivatives. But, of course, it will be also with the loss of one derivative from $\mathbf{g}$. This precludes one from using fixed-point argument to prove a local existence theorem. It seems that the only way to overcome the difficulty connected with the loss of derivatives phenomenon is the employment of the Nash-Moser technique (for hyperbolic problems see [2, 31, 21]). Recently, the Nash-Moser method was successfully used by Coulombel and Secchi 
[20] for two-dimensional (2D) compressible vortex sheets as well as for weakly stable shock waves (see also preparatory results in this direction in $[17,18,19]$ ). There is also a great hope to achieve a nonlinear local existence result for current-vortex sheets [45] by using Nash-Moser iterations.

If the boundary conditions (1.7b) are not dissipative, this does not, of course, mean that problem (1.7) is ill-posed. An alternative to the energy method was first suggested by Kreiss [27] for the strictly hyperbolic case. Kreiss has proved that problem (1.7) with constant ("frozen") coefficients obeys an a priori $L_{2, \eta}$-estimate with no loss of derivatives if and only if the boundary conditions satisfy the uniform Lopatinski condition [27] $\left(\|\cdot\|_{L_{2, \eta}}=\left\|e^{-\eta t}(\cdot)\right\|_{L_{2}}\right.$, and $\eta>0$ is sufficiently large). This estimate follows from a symmetrizer construction (Kreiss symmetrizer) and is carried over variable coefficients by using pseudodifferential calculus. That is, the uniform Lopatinski condition is the sharp algebraic criterion of strong well-posedness (well-posedness "with no loss of derivatives").

Later Kreiss' symmetrizer analysis was extended by Agranovich [1], Majda and Osher [35], and Majda [32] to hyperbolic systems satisfying a so-called block structure condition, which holds in particular for hyperbolic symmetrizable systems with constant multiplicities $[1,36]$. In [32] Majda extends the Kreiss theory to the case of Rankine-Hugoniot boundary conditions. Majda's approach has been then improved by Métivier [37] by using paradifferential calculus of Bony (see also discussion in section 4). And recently Métivier and Zumbrun [38] have extended the Kreiss-Majda theory to a class of hyperbolic symmetrizable systems with characteristics of variable multiplicities. These systems at points of variable multiplicity should satisfy some conditions [38] which hold in particular for the MHD system. Thus, if the symmetric hyperbolic system (1.6a) meets either the Agronovich-Majda-Osher block structure condition [1,35] or the conditions of Métivier and Zumbrun [38], then the linear problem (1.7) is strongly well-posed, provided that the boundary conditions (1.7b) satisfy the uniform Lopatinski condition. In this case a local existence theorem for the nonlinear problem (1.6) can be proved by analogy with the proofs in [33, 37].

At the same time, it should be noted that in practice the algebraic criterion given by the Lopatinski condition often cannot be tested analytically. Sometimes one succeeds to check it numerically (see [44]), but, frequently, numerical calculations can give only a very rough description of the condition for weak/strong well-posedness because either the domain of parameters for the constant coefficients linearized problem is unbounded or the number of these parameters is too big. Usually this happens for the case of Rankine-Hugoniot boundary conditions (see below), i.e., for shock waves or characteristic discontinuities. For example, such a situation takes place for compressible current-vortex sheets [45]. In this connection, there is no sense to reject at once the energy method as soon as the boundary conditions are not dissipative. The main purpose of the present paper is to formalize the so-called dissipative integrals technique (see [13]), which is a kind of "higher order" extension of the usual energy method. Especially, this technique turned out to be effective for shock waves in various hyperbolic models: gas dynamics, Landau's equations of superfluid, MHD, radiation hydrodynamics, etc. (see [13] and references therein). Let us now go on to the case of Rankine-Hugoniot boundary conditions.

1.2. Rankine-Hugoniot boundary conditions. Consider system (1.1) in the whole space $\mathbb{R}^{n}$. Let

$$
\Gamma(t)=\left\{x_{1}-f\left(t, \mathbf{x}^{\prime}\right)=0\right\}
$$


be a smooth hypersurface in $[0, T] \times \mathbb{R}^{n}$. We assume that $\Gamma(t)$ is a surface of strong discontinuity for solutions of (1.1). Let $\mathbf{U}(t, \mathbf{x})$ be a classical solution of (1.1) on either side of $\Gamma$. As is known, $\mathbf{U}$ is a weak solution of (1.1) if and only if the RankineHugoniot jump conditions hold at each point of $\Gamma$ :

$$
f_{t}\left[\mathcal{P}^{0}(\mathbf{U})\right]+\sum_{k=2}^{n} f_{x_{k}}\left[\mathcal{P}^{k}(\mathbf{U})\right]-\left[\mathcal{P}^{1}(\mathbf{U})\right]=0
$$

where $[a]=a^{+}-a^{-}=\left.a\right|_{x_{1}-f\left(t, \mathbf{x}^{\prime}\right)=+0}-\left.a\right|_{x_{1}-f\left(t, \mathbf{x}^{\prime}\right)=-0}$.

It should be noted that the initial-boundary-value problem for system (1.5) in the domains $\Omega^{ \pm}(t):=\left\{x_{1} \gtrless f\left(t, \mathbf{x}^{\prime}\right)\right\}$ with the boundary conditions (1.15) on the hypersurface $\Gamma(t)$ is a free-boundary-value problem. Indeed, the function $f\left(t, \mathbf{x}^{\prime}\right)$ defining $\Gamma$ is one of the unknowns of problem (1.5), (1.15) with the corresponding initial data

$$
\left.f\right|_{t=0}=f_{0} \quad \text { in } \mathbb{R}^{n-1},\left.\quad \mathbf{U}\right|_{t=0}=\mathbf{U}_{0} \quad \text { in } \Omega^{+}(0) \cup \Omega^{-}(0) .
$$

To work in fixed domains instead of the domains $\Omega^{ \pm}(t)$ we make the following change of variables:

$$
\widetilde{t}=t, \quad \widetilde{x}_{1}=x_{1}-f\left(t, \mathbf{x}^{\prime}\right), \quad \widetilde{\mathbf{x}}^{\prime}=\mathbf{x}^{\prime} .
$$

Then, $\widetilde{\mathbf{U}}(\widetilde{t}, \widetilde{\mathbf{x}}):=\mathbf{U}(t, \mathbf{x})$ is a smooth vector-function for $\widetilde{\mathbf{x}} \in \mathbb{R}_{ \pm}^{n}$, and the initialboundary-value problem (1.5), (1.15), (1.16) is reduced to the following problem (we omit tildes to simplify the notation):

$$
\begin{gathered}
L(\mathbf{U}, \mathbf{F}) \mathbf{U}=0 \quad \text { in }[0, T] \times\left(\mathbb{R}_{+}^{n} \cup \mathbb{R}_{-}^{n}\right), \\
B\left(\mathbf{U}^{+}, \mathbf{U}^{-}\right) \mathbf{F}-\left[\mathcal{P}^{1}(\mathbf{U})\right]=0 \quad \text { on }[0, T] \times\left\{x_{1}=0\right\} \times \mathbb{R}^{n-1}, \\
\left.\mathbf{U}\right|_{t=0}=\mathbf{U}_{0} \quad \text { in } \mathbb{R}_{+}^{n} \cup \mathbb{R}_{-}^{n},\left.\quad f\right|_{t=0}=f_{0} \quad \text { in } \mathbb{R}^{n-1}
\end{gathered}
$$

Here

$$
\begin{gathered}
L=L(\mathbf{U}, \mathbf{F})=A_{0}(\mathbf{U}) \partial_{t}+A_{\nu}(\mathbf{U}, \mathbf{F}) \partial_{1}+\sum_{k=2}^{n} A_{k}(\mathbf{U}) \partial_{k}, \\
\mathbf{F}=\mathbf{F}\left(t, \mathbf{x}^{\prime}\right)=\left(f_{t}, \mathbf{F}^{\prime}\right), \quad \mathbf{F}^{\prime}=\mathbf{F}^{\prime}\left(t, \mathbf{x}^{\prime}\right)=\nabla_{\mathbf{x}^{\prime}} f, \quad \nabla_{\mathbf{x}^{\prime}}=\left(\partial_{2}, \ldots, \partial_{n}\right), \\
A_{\nu}=A_{\nu}(\mathbf{U}, \mathbf{F})=\sum_{\alpha=0}^{n} \nu_{\alpha} A_{\alpha}=A_{1}(\mathbf{U})-f_{t} A_{0}(\mathbf{U})-\sum_{k=2}^{n} f_{x_{k}} A_{k}(\mathbf{U}),
\end{gathered}
$$

$\boldsymbol{\nu}=\left(\nu_{0}, \ldots, \nu_{n}\right)=\left(-f_{t}, \mathbf{N}\right)$ and $\mathbf{N}=\left(1,-\mathbf{F}^{\prime}\right)$ are, respectively, the space-time and space normal vectors to $\Gamma(t)$. The matrix $B=B\left(\mathbf{U}^{+}, \mathbf{U}^{-}\right)$is of order $N \times n$ and determined from the relation

$$
B\left(\mathbf{U}^{+}, \mathbf{U}^{-}\right) \mathbf{F}=f_{t}\left[\mathcal{P}^{0}(\mathbf{U})\right]+\sum_{k=2}^{n} f_{x_{k}}\left[\mathcal{P}^{k}(\mathbf{U})\right], \quad \mathbf{U}^{ \pm}:=\left.\mathbf{U}\right|_{x_{1}= \pm 0}
$$
form

After straightening of variables above the divergent constraints (1.3) take the

$$
\operatorname{div} \boldsymbol{\psi}_{j}\left(\mathbf{U}, \mathbf{F}^{\prime}\right)=0 \quad \text { in }[0, T] \times\left(\mathbb{R}_{+}^{n} \cup \mathbb{R}_{-}^{n}\right), \quad j=\overline{1, K},
$$


where $\boldsymbol{\psi}_{j}=\boldsymbol{\psi}_{j}\left(\mathbf{U}, \mathbf{F}^{\prime}\right)=\left(\left(\boldsymbol{\Psi}_{j}, \mathbf{N}\right), \Psi_{j}^{2}, \ldots, \Psi_{j}^{n}\right)$. Moreover, solutions of problem (1.17) should satisfy the jump conditions

$$
\left[\left(\mathbf{\Psi}_{j}(\mathbf{U}), \mathbf{N}\right)\right]=0 \quad \text { on }[0, T] \times\left\{x_{1}=0\right\} \times \mathbb{R}^{n-1}, \quad j=\overline{1, K},
$$

coming from (1.3). At the same time, taking into account Assumption 1.1 and Remark 1.1, it is natural to make the following assumption that should be true for all the physically relevant models.

ASSUMPTION 1.2. The divergent constraints (1.18) are the restrictions on the initial data (1.17c), i.e., if (1.18) are satisfied initially, they hold for all $t>0$. Equations (1.17b) and (1.19) form a system of $N$ independent boundary conditions.

To clarify Assumption 1.2 we can refer, for example, to MHD. Namely, in MHD the jump condition $f_{t}[(\mathbf{H}, \mathbf{N})]=0$ contained in the corresponding main system, $(1.17 \mathrm{~b})$, is implied by the equation $[(\mathbf{H}, \mathbf{N})]=0$ coming from the divergent constraint $\operatorname{div} \mathbf{H}=0$. That is, in MHD the number of independent Rankine-Hugoniot boundary conditions is equal to the number of conservation laws.

To prove the existence of solutions with a surface of strong discontinuity $\Gamma(t)$ for the system of hyperbolic conservation laws (1.1) one needs to reply to the following question: does there exist a solution $(\mathbf{U}, f)$ to problem (1.17) at least locally in time? The necessary (but not sufficient) condition for this is that the hyperbolic problem (1.17) has the correct number of boundary conditions in (1.17b). In this connection, it should be noted that, in contrast with the standard boundary conditions (1.6b), one of the conditions in (1.17b) is needed for determining the function $f\left(t, \mathbf{x}^{\prime}\right)$. For noncharacteristic discontinuities, i.e., shock waves, the plane $x_{1}=0$ is not a characteristic boundary for system (1.17). That is, the boundary matrix $A_{\nu}$ is nonsingular at $x_{1}=0: \operatorname{det} A_{\nu}^{ \pm} \neq 0$, where $A_{\nu}^{ \pm}=\left.A_{\nu}\right|_{x_{1}= \pm 0}$. As is known, for shock waves the correct number of Rankine-Hugoniot boundary conditions is guaranteed by the Lax shock conditions. They can be conveniently written in terms of the eigenvalues of the boundary matrix $A_{\nu}$ :

$$
\lambda_{k}\left(A_{\nu}^{+}\right)<0<\lambda_{k}\left(A_{\nu}^{-}\right), \quad \lambda_{k-1}\left(A_{\nu}^{-}\right)<0<\lambda_{k+1}\left(A_{\nu}^{+}\right),
$$

where $\lambda_{i}\left(A_{\nu}^{ \pm}\right)\left(i=\overline{1, N}, \lambda_{1} \leq \ldots \leq \lambda_{N}\right)$ are the eigenvalues of the matrices $A_{\nu}^{ \pm}$and $k$ is a fixed integer number, $1 \leq k \leq N$ (an associated shock wave is called $k$-shock), $\lambda_{0}:=-\infty, \lambda_{N+1}:=+\infty$.

Let $\left(\widehat{\mathbf{U}}(t, \mathbf{x}), \hat{f}\left(t, \mathbf{x}^{\prime}\right)\right)$ be a given vector-function, where $\widehat{\mathbf{U}}$ is supposed to be smooth for $\mathbf{x} \in \mathbb{R}_{ \pm}^{n}$. Then the linearization of (1.17) results in the following variable coefficients problem for determining small perturbations $(\delta \mathbf{U}, \delta f)$ (below we drop $\delta$ ):

$$
\begin{array}{r}
L(\widehat{\mathbf{U}}, \widehat{\mathbf{F}}) \mathbf{U}+\widehat{C} \mathbf{U}=\{L(\widehat{\mathbf{U}}, \widehat{\mathbf{F}}) f\} \widehat{\mathbf{U}}_{x_{1}} \quad \text { in }[0, T] \times\left(\mathbb{R}_{+}^{n} \cup \mathbb{R}_{-}^{n}\right), \\
B\left(\widehat{\mathbf{U}}^{+}, \widehat{\mathbf{U}}^{-}\right) \mathbf{F}-\left[S^{-1}(\widehat{\mathbf{U}}) A_{\nu}(\widehat{\mathbf{U}}, \widehat{\mathbf{F}}) \mathbf{U}\right]=0 \quad \text { on }[0, T] \times\left\{x_{1}=0\right\} \times \mathbb{R}^{n-1},
\end{array}
$$

and the initial data for the perturbation $(\mathbf{U}, f)$ coincide with $(1.17 \mathrm{c})$. Here, $\widehat{\mathbf{F}}=$ $\left(\hat{f}_{t}, \widehat{\mathbf{F}}^{\prime}\right), \widehat{\mathbf{F}}^{\prime}=\nabla_{\mathbf{x}^{\prime}} \hat{f}$, the matrix $\widehat{C}=\widehat{C}\left(\widehat{\mathbf{U}}, \widehat{\mathbf{U}}_{t}, \nabla \widehat{\mathbf{U}}, \widehat{\mathbf{F}}\right)$ is determined as follows:

$$
\widehat{C} \mathbf{U}=\left(\mathbf{U}, \nabla_{u} A_{0}(\widehat{\mathbf{U}})\right) \widehat{\mathbf{U}}_{t}+\left(\mathbf{U}, \nabla_{u} A_{\nu}(\widehat{\mathbf{U}}, \widehat{\mathbf{F}})\right) \widehat{\mathbf{U}}_{x_{1}}+\sum_{k=2}^{n}\left(\mathbf{U}, \nabla_{u} A_{k}(\widehat{\mathbf{U}})\right) \widehat{\mathbf{U}}_{x_{k}},
$$

$\left(\mathbf{U}, \nabla_{u}\right):=\sum_{i=1}^{N} u_{i} \partial / \partial u_{i}$. Recall that $S(\mathbf{U})$ is the Friedrichs symmetrizer mentioned above, i.e., $S^{-1} A_{\nu}=B_{\nu}$, where $B_{\nu}=\sum_{\alpha=0}^{n} \nu_{\alpha} B_{\alpha}$. Problem (1.21a), (1.17c) is the 
genuine linearization of (1.17) in the sense that we keep all the lower order terms in (1.21a).

It should be noted that the differential operator in system $(1.21 \mathrm{a})$ is a first order operator in $f$. This fact can give some trouble in the application of the energy method to (1.21a). To avoid this difficulty we make the change of unknowns (see [2])

$$
\overline{\mathbf{U}}=\mathbf{U}-f \widehat{\mathbf{U}}_{x_{1}} .
$$

In terms of the "good unknown" (1.22) problem (1.21a) takes the form

$$
\begin{aligned}
& L(\widehat{\mathbf{U}}, \widehat{\mathbf{F}}) \overline{\mathbf{U}}+\widehat{C} \overline{\mathbf{U}}+f \partial_{1}\{L(\widehat{\mathbf{U}}, \widehat{\mathbf{F}}) \widehat{\mathbf{U}}\}=0 \quad \text { in }[0, T] \times\left(\mathbb{R}_{+}^{n} \cup \mathbb{R}_{-}^{n}\right), \\
& B\left(\widehat{\mathbf{U}}^{+}, \widehat{\mathbf{U}}^{-}\right) \mathbf{F}-\left[S^{-1}(\widehat{\mathbf{U}}) A_{\nu}(\widehat{\mathbf{U}}, \widehat{\mathbf{F}}) \overline{\mathbf{U}}\right] \\
& \quad=-f\left[S^{-1}(\widehat{\mathbf{U}}) A_{\nu}(\widehat{\mathbf{U}}, \widehat{\mathbf{F}}) \widehat{\mathbf{U}}_{x_{1}}\right] \quad \text { on }[0, T] \times\left\{x_{1}=0\right\} \times \mathbb{R}^{n-1} .
\end{aligned}
$$

Actually, to prove a local existence theorem for the nonlinear problem (1.17) with strictly dissipative boundary conditions or having a strictly dissipative $p$-symmetrizer (see section 2), it is not necessary to consider the genuine linearization (1.21a). It is enough to keep only the principal part of the linearized equations, i.e., one can drop the lower order terms in (1.23). At the same time, for the case when the loss of derivatives takes place it needs to perform genuine linearization ("to find a differential") for the purpose of a possible use of the Nash-Moser method (see discussion in Remark 1.2). The linearized equations associated to (1.17a), (1.17b) and obtained by dropping the lower order terms in (1.23) read:

$$
\begin{gathered}
L(\widehat{\mathbf{U}}, \widehat{\mathbf{F}}) \mathbf{U}=\mathbf{f} \quad \text { in }[0, T] \times\left(\mathbb{R}_{+}^{n} \cup \mathbb{R}_{-}^{n}\right), \\
B\left(\widehat{\mathbf{U}}^{+}, \widehat{\mathbf{U}}^{-}\right) \mathbf{F}-\left[S^{-1}(\widehat{\mathbf{U}}) A_{\nu}(\widehat{\mathbf{U}}, \widehat{\mathbf{F}}) \mathbf{U}\right]=\mathbf{g} \quad \text { on }[0, T] \times\left\{x_{1}=0\right\} \times \mathbb{R}^{n-1} .
\end{gathered}
$$

Here we introduce the source terms $\mathbf{f}(t, \mathbf{x})$ and $\mathbf{g}\left(t, \mathbf{x}^{\prime}\right)$, where $\mathbf{f}(t, \mathbf{x})=\mathbf{f}^{ \pm}(t, \mathbf{x})$ for $\mathrm{x} \in \mathbb{R}_{ \pm}^{n}$.

For problem (1.24) the definitions of dissipative and strictly dissipative boundary conditions are analogous to those in (1.12) and (1.11). In particular, the homogenous boundary conditions $(1.24 \mathrm{~b})(\mathbf{g}=0)$ are dissipative if $-\left.\left[\left(A_{\nu} \mathbf{U}, \mathbf{U}\right)\right]\right|_{x_{1}=0} \geq 0$ for all $\mathbf{U}$ satisfying (1.24b). Assuming that the front can be eliminated, i.e., the vector-function $\mathbf{F}$ can be expressed through $\mathbf{U}^{+}, \mathbf{U}^{-}$, and $\mathbf{g}$ (see section 3 for more details), for shock waves the counterparts of estimates (1.14) and (1.13) are the following:

$$
\begin{aligned}
& \|f\|_{W_{2}^{1}\left([0, T] \times \mathbb{R}^{n-1}\right)}+\sum_{ \pm}\|\mathbf{U}(t)\|_{W_{2}^{1}\left(\mathbb{R}_{ \pm}^{n}\right)} \leq C\left\{\left\|f_{0}\right\|_{W_{2}^{1}\left(\mathbb{R}^{n-1}\right)}\right. \\
& \left.\quad+\sum_{ \pm}\left\{\left\|\mathbf{U}_{0}\right\|_{W_{2}^{1}\left(\mathbb{R}_{ \pm}^{n}\right)}+\left\|\mathbf{f}^{ \pm}\right\|_{W_{2}^{1}\left([0, T] \times \mathbb{R}_{ \pm}^{n}\right)}\right\}+\|\mathbf{g}\|_{W_{2}^{2}\left([0, T] \times \mathbb{R}^{n-1}\right)}\right\}
\end{aligned}
$$

(for dissipative boundary conditions),

$$
\begin{aligned}
& \|f\|_{W_{2}^{1}\left([0, T] \times \mathbb{R}^{n-1}\right)}+\sum_{ \pm}\|\mathbf{U}(t)\|_{L_{2}\left(\mathbb{R}_{ \pm}^{n}\right)} \leq C\left\{\left\|f_{0}\right\|_{W_{2}^{1}\left(\mathbb{R}^{n-1}\right)}\right. \\
& \left.\quad+\sum_{ \pm}\left\{\left\|\mathbf{U}_{0}\right\|_{L_{2}\left(\mathbb{R}_{ \pm}^{n}\right)}+\left\|\mathbf{f}^{ \pm}\right\|_{L_{2}\left([0, T] \times \mathbb{R}_{ \pm}^{n}\right)}\right\}+\|\mathbf{g}\|_{L_{2}\left([0, T] \times \mathbb{R}^{n-1}\right)}\right\}
\end{aligned}
$$

(for strictly dissipative boundary conditions). 
Since the original nonlinear problem (1.17) is a reduced free-boundary-value problem, we should gain the "additional derivative" for the front perturbation $f$ (cf. (1.26)) to use then fixed-point argument. In this sense the a priori estimate (1.25) indicates the loss of one derivative not only from the source term $\mathbf{g}$ but also from the front $f$. At the same time, Rankine-Hugoniot boundary conditions are usually not dissipative (not to mention strict dissipativity). Nevertheless, the energy method can be still applied to problem (1.17) under certain circumstances. The main idea is to obtain from the linearized problem a problem for higher order derivatives of $\mathbf{U}$ so that this problem has dissipative (or strictly dissipative) boundary conditions. Such an idea was first realized by Blokhin [7,9] for shock waves in gas dynamics. In the next section we formalize this idea by introducing the notations of dissipative and strictly dissipative $p$-symmetrizers and give concrete examples of $p$-symmetrizers. In section 3 we consider the case of constant coefficients and deduce a priori estimates for Lax shock waves whose linearized problems have a $p$-symmetrizer. In section 4 we carry these estimates over variable coefficients and outline the proof of the local existence theorem for the original nonlinear problem (1.17), provided that the corresponding constant coefficients linearized problem has a strictly dissipative $p$-symmetrizer. For all the linear results obtained earlier by the dissipative integrals technique for shock waves in various concrete models (see [13] and references therein) this enables one to conclude the local existence of shock-front solutions of the corresponding nonlinear systems. Eventually, in section 5 we make concluding remarks and discuss open problems.

2. Dissipative $\boldsymbol{p}$-symmetrizers: Definition and examples. We introduce the notations of dissipative and strictly dissipative $p$-symmetrizers for linear hyperbolic initial-boundary-value problems with constant coefficients. We give the definition of these notations for the case of Rankine-Hugoniot boundary conditions. The corresponding definition for the earthier case of standard boundary conditions (cf. $(1.6 \mathrm{~b})$ ) is given analogously and does not need a separate treatment.

The constant coefficients linearized problem for planar discontinuities is of decisive importance for the subsequent variable coefficients and nonlinear analysis. For planar discontinuities $\hat{f}\left(t, \mathbf{x}^{\prime}\right)$ is a linear function:

$$
\hat{f}\left(t, \mathbf{x}^{\prime}\right)=\sigma t+\left(\boldsymbol{\sigma}^{\prime}, \mathbf{x}^{\prime}\right), \quad \boldsymbol{\sigma}=\left(\sigma, \boldsymbol{\sigma}^{\prime}\right) \in \mathbb{R}^{n} .
$$

For the case of a piecewise constant solution,

$$
\widehat{\mathbf{U}}= \begin{cases}\widehat{\mathbf{U}}^{+}, & x_{1}>\sigma t+\left(\boldsymbol{\sigma}^{\prime}, \mathbf{x}^{\prime}\right), \\ \widehat{\mathbf{U}}^{-}, & x_{1}<\sigma t+\left(\boldsymbol{\sigma}^{\prime}, \mathbf{x}^{\prime}\right),\end{cases}
$$

equations (1.24) have constant ("frozen") coefficients:

$$
\begin{gathered}
L\left(\widehat{\mathbf{U}}^{ \pm}, \boldsymbol{\sigma}\right) \mathbf{U}=\mathbf{f}^{ \pm} \quad \text { for } \mathbf{x} \in \mathbb{R}_{ \pm}^{n}, \\
\widehat{B} \mathbf{F}-\left[\widehat{S}^{-1} \widehat{A}_{\nu} \mathbf{U}\right]=\mathbf{g} \quad \text { for } x_{1}=0,
\end{gathered}
$$

where $\widehat{B}=B\left(\widehat{\mathbf{U}}^{+}, \widehat{\mathbf{U}}^{-}\right), \widehat{S}^{ \pm}=S\left(\widehat{\mathbf{U}}^{ \pm}\right), \widehat{A}_{\nu}^{ \pm}=A_{\nu}\left(\widehat{\mathbf{U}}^{ \pm}, \boldsymbol{\sigma}\right), \widehat{A}_{\alpha}^{ \pm}=A_{\alpha}\left(\widehat{\mathbf{U}}^{ \pm}\right)$are constant coefficients matrices, and $\left[\widehat{S}^{-1} \widehat{A}_{\nu} \mathbf{U}\right]=\left(\widehat{S}^{+}\right)^{-1} \widehat{A}_{\nu}^{+} \mathbf{U}^{+}-\left(\widehat{S}^{-}\right)^{-1} \widehat{A}_{\nu}^{-} \mathbf{U}^{-}$, etc.

Assumption 2.1. The functions $\Psi_{j}^{i}(\mathbf{U})$ in (1.3) are linear.

Assumption 2.1 is satisfied for most examples of constrained hyperbolic systems we know. At the same time, this assumption is made only for simplicity of arguments 
below and can be easily removed. Assuming the linearity of $\Psi_{j}^{i}(\mathbf{U})$, the linearized constraints (1.18) after the change of unknowns (1.22) (below we drop the bars) read:

$$
\operatorname{div} \boldsymbol{\psi}_{j}=0, \quad \mathbf{x} \in \mathbb{R}_{ \pm}^{n}, \quad j=\overline{1, K},
$$

where $\boldsymbol{\psi}_{j}=\boldsymbol{\psi}_{j}\left(\mathbf{U}, \widehat{\mathbf{F}}^{\prime}\right)=\left(\left(\boldsymbol{\Psi}_{j}, \widehat{\mathbf{N}}\right), \Psi_{j}^{2}, \ldots, \Psi_{j}^{n}\right), \widehat{\mathbf{N}}=\left(1,-\widehat{\mathbf{F}}^{\prime}\right)$. For the case of constant coefficients $(2.2), \widehat{\mathbf{N}}=\left(1,-\boldsymbol{\sigma}^{\prime}\right)$.

2.1. The main definition. Given a nonnegative integer number $p$, we introduce the notation

$$
\mathbf{W}_{p}:=\left(\partial^{\alpha^{1}} \mathbf{U}, \ldots, \partial^{\alpha^{d}} \mathbf{U}\right)
$$

with

$$
d=C_{n+p}^{p}, \quad\left|\alpha^{i}\right|=p, \quad i=\overline{1, d}, \quad \alpha^{i} \neq \alpha^{j} \quad \text { for } i \neq j,
$$

where $\partial^{\alpha}:=\partial_{t}^{\alpha_{0}} \partial_{1}^{\alpha_{1}} \ldots \partial_{n}^{\alpha_{n}}, \alpha=\left(\alpha_{0}, \ldots, \alpha_{n}\right)$. In particular, $\mathbf{W}_{0}=\mathbf{U}, \mathbf{W}_{1}=$ $\left(\mathbf{U}_{t}, \mathbf{U}_{x_{1}}, \ldots, \mathbf{U}_{x_{n}}\right), \mathbf{W}_{2}=\left(\mathbf{U}_{t t}, \mathbf{U}_{t x_{1}}, \ldots, \mathbf{U}_{x_{n-1} x_{n}}, \mathbf{U}_{x_{n} x_{n}}\right)$. Below we will usually omit the index $p$, i.e., $\mathbf{W}:=\mathbf{W}_{p}$.

Differentiating systems $(2.2 \mathrm{a})$ (if $p \neq 0$ ) and taking into account relations (2.3), one gets

$$
P^{ \pm} \widetilde{L}\left(\widehat{\mathbf{U}}^{ \pm}, \boldsymbol{\sigma}\right) \mathbf{W}+\sum_{j=1}^{K} \sum_{|\alpha|=p} \mathbf{R}_{j, \alpha}^{ \pm} \operatorname{div}\left(\boldsymbol{\psi}_{j}\left(\partial^{\alpha} \mathbf{U}, \boldsymbol{\sigma}^{\prime}\right)\right)=P^{ \pm} \widetilde{\mathbf{f}}^{ \pm}, \quad \mathbf{x} \in \mathbb{R}_{ \pm}^{n}
$$

where

$$
\widetilde{L}\left(\widehat{\mathbf{U}}^{ \pm}, \boldsymbol{\sigma}\right)=I_{d} \otimes L\left(\widehat{\mathbf{U}}^{ \pm}, \boldsymbol{\sigma}\right), \quad \widetilde{\mathbf{f}}^{ \pm}=\left(\partial^{\alpha^{1}} \mathbf{f}^{ \pm}, \ldots, \partial^{\alpha^{d}} \mathbf{f}^{ \pm}\right)
$$

$\mathbf{R}_{j, \alpha}^{ \pm}=\mathbf{R}_{j, \alpha}\left(\widehat{\mathbf{U}}^{ \pm}, \boldsymbol{\sigma}\right)$ and $P^{ \pm}=P\left(\widehat{\mathbf{U}}^{ \pm}, \boldsymbol{\sigma}\right)$ are, respectively, vectors and nonsingular matrices of order $N d$. Here and below the subscript in $I_{j}$ indicates the order of the unit matrix $I_{j}=I$ (sometimes we omit it). Systems (2.4) can be rewritten as follows:

$$
\mathcal{L}\left(\widehat{\mathbf{U}}^{ \pm}, \boldsymbol{\sigma}\right) \mathbf{W}=\mathcal{F}^{ \pm}, \quad \mathbf{x} \in \mathbb{R}_{ \pm}^{n}
$$

where

$$
\mathcal{L}=\mathcal{L}\left(\widehat{\mathbf{U}}^{ \pm}, \boldsymbol{\sigma}\right)=\widehat{\mathcal{A}}_{0}^{ \pm} \partial_{t}+\sum_{j=1}^{n} \widehat{\mathcal{A}}_{j}^{ \pm} \partial_{j}, \quad \mathcal{F}^{ \pm}=\mathcal{F}^{ \pm}\left(\widehat{\mathbf{U}}^{ \pm}, \boldsymbol{\sigma}\right)=P^{ \pm} \widetilde{\mathbf{f}}^{ \pm}
$$

and $\widehat{\mathcal{A}}_{i}^{ \pm}=\mathcal{A}_{i}\left(\widehat{\mathbf{U}}^{ \pm}, \boldsymbol{\sigma}\right), i=\overline{0, n}$, are matrices of order $N d$ (their explicit form is determined from $(2.4))$.

Systems (2.5) are a kind of "secondary higher order" symmetrization of the symmetric systems (2.2a) if the matrices $\widehat{\mathcal{A}}_{i}^{ \pm}$are again symmetric. Moreover, to make these matrices symmetric when writing out (2.5) one can take into account the trivial relations like

$$
\partial_{i} \partial_{j} \mathbf{U}=\partial_{j} \partial_{i} \mathbf{U}, \quad \partial_{i} \partial_{j} \partial_{k} \mathbf{U}=\partial_{j} \partial_{i} \partial_{k} \mathbf{U}=\ldots=\partial_{k} \partial_{j} \partial_{i} \mathbf{U}, \quad \text { etc. },
$$

which follow from the smoothness hypothesis (in $\mathbb{R}_{ \pm}^{n}$ ). Clearly, systems (2.5) are not uniquely written out. It follows that a corresponding $p$-symmetrizer (see the definition below) is not uniquely determined and, in principle, the problem can have different $p$-symmetrizers. It is however quite natural and does not, of course, imply the nonuniqueness of solutions to the problem. 
The boundary conditions for systems (2.5) are obtained by the tangential differentiation (with respect to $t$ and $\mathbf{x}^{\prime}$ ) of conditions $(2.2 \mathrm{~b}$ ), and, furthermore, systems (2.4) themselves differentiated $p-1$ times and considered at $x_{1}=0$ can be used as boundary conditions. Unfortunately, the boundary conditions are difficult to present in a concrete form, but it is clear that the right-hand parts in them depend on $\partial_{t, \mathrm{x}^{\prime}}^{\alpha} \mathbf{g}$, with $|\alpha|=p$, and $\left.\partial^{\beta} \mathbf{f}^{ \pm}\right|_{x_{1}= \pm 0}$, with $|\beta|=p-1$, where $\partial_{t, \mathbf{x}^{\prime}}^{\alpha}:=\partial_{t}^{\alpha_{0}} \partial_{2}^{\alpha_{2}} \cdots \partial_{n}^{\alpha_{n}}$, $\alpha=\left(\alpha_{0}, \alpha_{2}, \ldots, \alpha_{n}\right)$. The corresponding vector of right-hand parts formed by $\partial_{t, \mathrm{x}^{\mathrm{x}}}^{\alpha} \mathbf{g}$ and $\left.\partial^{\beta} \mathbf{f}^{ \pm}\right|_{x_{1}= \pm 0}$ is below denoted by $\mathcal{G}$.

We are now in a position to give the definition of the (strictly) dissipative $p$ symmetrizer.

Definition 2.1. The set of matrices and vectors

$$
\mathbb{S}=\mathbb{S}\left(\widehat{\mathbf{U}}^{+}, \widehat{\mathbf{U}}^{-}, \boldsymbol{\sigma}\right):=\left\{P^{+}, P^{-},\left\{\mathbf{R}_{j, \alpha}^{+}\right\}_{j=\overline{1, K},|\alpha|=p},\left\{\mathbf{R}_{j, \alpha}^{-}\right\}_{j=\overline{1, K},|\alpha|=p}\right\}
$$

$\left(\mathbb{S}:=\left\{P^{+}, P^{-}\right\}\right.$if system (1.1) has no divergent constraints) is called the dissipative p-symmetrizer of problem (2.2) if the matrices $\widehat{\mathcal{A}}_{i}^{ \pm}$in (2.5) are symmetric and there is an open subset $D$ of the state space $G \subset \mathbb{R}^{N} \times \mathbb{R}^{N} \times \mathbb{R}^{n}$, a constant matrix $B$, and a constant $\gamma$ such that

$$
\widehat{\mathcal{A}}_{0}^{+}>0, \quad \widehat{\mathcal{A}}_{0}^{-}>0
$$

and (cf. (1.12))

$$
-\left.\left[\left(\widehat{\mathcal{A}_{1}} \mathbf{W}, \mathbf{W}\right)\right]\right|_{x_{1}=0} \geq(B \mathcal{G}, \widetilde{\mathbf{W}})-\gamma|\mathcal{G}|^{2}
$$

for all $\left(\widehat{\mathbf{U}}^{+}, \widehat{\mathbf{U}}^{-}, \boldsymbol{\sigma}\right) \in D$ and all $\mathbf{W}$ satisfying the boundary conditions for systems (2.5), where $\widetilde{\mathbf{W}}=\left(\widetilde{\mathbf{W}}^{+}, \widetilde{\mathbf{W}}^{-}\right)$and $\widetilde{\mathbf{W}}^{ \pm}$is the projection of $\mathbf{W}^{ \pm}$orthogonal to ker $\widehat{\mathcal{A}}_{1}^{ \pm}$ (for shock waves $\widetilde{\mathbf{W}}^{ \pm}=\mathbf{W}^{ \pm}=\left.\mathbf{W}\right|_{x_{1}= \pm 0}$ ).

The set $\mathbb{S}$ is called the strictly dissipative p-symmetrizer of problem (2.2) if it is a dissipative p-symmetrizer of this problem and there is a fixed constant $\delta>0$ such that

$$
-\left.\left[\left(\widehat{\mathcal{A}_{1}} \mathbf{W}, \mathbf{W}\right)\right]\right|_{x_{1}=0} \geq \delta|\widetilde{\mathbf{W}}|^{2}-\delta^{-1}|\mathcal{G}|^{2} .
$$

Remark 2.1. For 1-shocks $\widehat{A}_{\nu}^{-}>0$ (cf. (1.20)) and, therefore, the strictly dissipative $p$-symmetrizer can be taken in the form

$$
\mathbb{S}=\left\{P^{+}, \gamma I,\left\{\mathbf{R}_{j, \alpha}^{+}\right\}_{j=1, K,|\alpha|=p}, 0, \ldots, 0\right\},
$$

where the constant $\gamma>0$ is large enough, $P^{+}, \mathbf{R}_{j, \alpha}^{+}$are such that the matrices $\widehat{\mathcal{A}}_{\alpha}^{+}$ are symmetric, $\widehat{\mathcal{A}}_{0}^{+}>0$, and the relaxed condition (2.8),

$$
-\left.\left(\widehat{\mathcal{A}}_{1}^{+} \mathbf{W}, \mathbf{W}\right)\right|_{x_{1}=+0} \geq \delta\left|\mathbf{W}^{+}\right|^{2}-\delta^{-1}\left\{|\mathcal{G}|^{2}+\left|\mathbf{W}^{-}\right|^{2}\right\},
$$

is fulfilled. Thanks to the choice of $\gamma$ and the condition $\widehat{\mathcal{A}}_{1}^{-}=\gamma\left(I_{d} \otimes \widehat{A}_{\nu}^{-}\right)>0$, the last inequality implies (2.8) with an appropriate (and different) $\delta$. That is, systems (2.5) are symmetric hyperbolic and the boundary conditions for them are strictly dissipative (cf. (1.11)).

Remark 2.2. The definition of the $p$-symmetrizer for the case of standard boundary conditions is analogous to Definition 2.1. Note only that for problem (1.7) (with 
"frozen" coefficients) the $p$-symmetrizer has the form $\mathbb{S}=\left\{P,\left\{\mathbf{R}_{j, \alpha}\right\}_{j=\overline{1, K},|\alpha|=p}\right\}$, where $P$ is a nonsingular matrix of order $N C_{n+p}^{p}$, etc.

We now give some concrete examples of dissipative and strictly dissipative $p$ symmetrizers. Without loss of generality we will consider homogenous interior equations and homogenous boundary conditions.

2.2. Example 1: The wave equation. Consider the initial-boundary-value problem in the half-plane $\mathbb{R}_{+}^{2}$ for the $2 \mathrm{D}$ wave equation:

$$
\begin{gathered}
u_{t t}=u_{x_{1} x_{1}}+u_{x_{2} x_{2}} \quad \text { for } x_{1}>0, \\
u_{t}+a u_{x_{1}}+b u_{x_{2}}=0 \quad \text { for } x_{1}=0,
\end{gathered}
$$

where $a$ and $b$ are real constants. As is known, the boundary conditions (2.9b) satisfy the uniform Lopatinski condition in the half-strip

$$
|b|<1, \quad a<0 .
$$

Problem (2.9) is easily reduced in the following problem for a symmetric hyperbolic system for the vector $\mathbf{U}=\left(u_{1}, u_{2}, u_{3}\right)=\left(u_{t}, u_{x_{1}}, u_{x_{2}}\right)$ :

$$
\begin{gathered}
\mathbf{U}_{t}+A_{1} \mathbf{U}_{x_{1}}+A_{2} \mathbf{U}_{x_{2}}=0 \quad \text { for } x_{1}>0, \\
M \mathbf{U}=0 \quad \text { for } x_{1}=0,
\end{gathered}
$$

with

$$
A_{1}=\left(\begin{array}{ccc}
0 & -1 & 0 \\
-1 & 0 & 0 \\
0 & 0 & 0
\end{array}\right), \quad A_{2}=\left(\begin{array}{ccc}
0 & 0 & -1 \\
0 & 0 & 0 \\
-1 & 0 & 0
\end{array}\right), \quad M=\left(\begin{array}{lll}
1 & a & b
\end{array}\right) .
$$

In terms of the components of the vector $\mathbf{U}$ the trivial relation $u_{x_{1} x_{2}}=u_{x_{2} x_{1}}$ satisfied by classical solutions of (2.9) reads:

$$
\operatorname{div} \boldsymbol{\Psi}(\mathbf{U})=0, \quad \Psi=\left(u_{3},-u_{2}\right) .
$$

Let us now forget about the connection between problems (2.9) and (2.11) (it is only important that for (2.11) the uniform Lopatinski condition is also given by (2.10)). Then (2.12) should be considered as a divergent constraint for the initial data for (2.11). Indeed, one can easily show that if (2.12) is satisfied initially, it holds for solutions of (2.11a) for all $t>0$.

We now prove that (2.11) has a strictly dissipative 0 -symmetrizer which can be taken in the form $\mathbb{S}=\{P, \mathbf{R}\}$, with

$$
P=\left(\begin{array}{ccc}
p_{1} & p_{2} & p_{3} \\
p_{2} & p_{1} & 0 \\
p_{3} & 0 & p_{1}
\end{array}\right), \quad R=\left(\begin{array}{c}
0 \\
-p_{3} \\
p_{2}
\end{array}\right), \quad p_{i} \in \mathbb{R}
$$

and the parameter domain $D$ (see Definition 2.1 and Remark 2.2) coincides with the the domain of fulfillment of the uniform Lopatinski condition, (2.10). Indeed, applying $\mathbb{S}$ to $(2.11),(2.12)$ leads to the system

$$
P \mathbf{U}_{t}+P A_{1} \mathbf{U}_{x_{1}}+P A_{2} \mathbf{U}_{x_{2}}+\mathbf{R} \operatorname{div} \boldsymbol{\Psi}=\mathcal{A}_{0} \mathbf{U}_{t}+\mathcal{A}_{1} \mathbf{U}_{x_{1}}+\mathcal{A}_{2} \mathbf{U}_{x_{2}}=0,
$$


where $\mathcal{A}_{0}=P>0$ if $p_{1}>0$ and $p_{1}^{2}-p_{2}^{2}-p_{3}^{2}>0$. Also,

$$
\mathcal{A}_{1}=\left(\begin{array}{ccc}
-p_{2} & -p_{1} & 0 \\
-p_{1} & -p_{2} & -p_{3} \\
0 & -p_{3} & p_{2}
\end{array}\right), \quad \mathcal{A}_{2}=\left(\begin{array}{ccc}
-p_{3} & 0 & -p_{1} \\
0 & p_{3} & -p_{2} \\
-p_{1} & -p_{2} & -p_{3}
\end{array}\right) .
$$

Note that $\mathcal{A}_{0}=\mathcal{T}^{*}\left\{I_{2} \otimes \mathcal{H}\right\} \mathcal{T}\left(\mathcal{A}_{0}>0\right.$ if $\left.\mathcal{H}>0\right)$,

$$
\begin{array}{cc}
\mathcal{A}_{1}=\mathcal{T}^{*}\left\{\left(\begin{array}{ll}
0 & 1 \\
1 & 0
\end{array}\right) \otimes \mathcal{H}\right\} \mathcal{T}, & \mathcal{A}_{2}=\mathcal{T}^{*}\left\{\left(\begin{array}{cc}
1 & 0 \\
0 & -1
\end{array}\right) \otimes \mathcal{H}\right\} \mathcal{T}, \\
\mathcal{T}=\frac{1}{\sqrt{2}}\left(\begin{array}{ccc}
1 & 0 & -1 \\
0 & -1 & 0 \\
0 & -1 & 0 \\
1 & 0 & 1
\end{array}\right), \quad \mathcal{H}=\left(\begin{array}{cc}
p_{1}-p_{3} & -p_{2} \\
-p_{2} & p_{1}+p_{3}
\end{array}\right) .
\end{array}
$$

Omitting calculations, one has

$$
-\left.\left(\mathcal{A}_{1} \mathbf{U}, \mathbf{U}\right)\right|_{x_{1}=0}=-\left.\left(\left\{\mathcal{S}^{*} \mathcal{H}+\mathcal{H S}\right\} \mathbf{V}_{2}, \mathbf{V}_{2}\right)\right|_{x_{1}=0}
$$

where

$$
\mathbf{V}=\mathcal{T} \mathbf{U}=\left(\begin{array}{c}
\mathbf{V}_{1} \\
\mathbf{V}_{2}
\end{array}\right),\left.\quad \mathbf{V}_{1}\right|_{x_{1}=0}=\left.\mathcal{S} \mathbf{V}_{2}\right|_{x_{1}=0}, \quad \mathcal{S}=\left(\begin{array}{cc}
\frac{2 a}{1-b} & \frac{b+1}{b-1} \\
1 & 0
\end{array}\right)
$$

All the eigenvalues of the matrix $\mathcal{S}$ lie in the left half-plane $\left(\Re \lambda_{i}(\mathcal{S})<0\right)$, provided that the uniform Lopatinski condition (2.10) holds. In this case the Lyapunov matrix equation [5]

$$
\mathcal{S}^{*} \mathcal{H}+\mathcal{H S}=-G
$$

has a unique solution $\mathcal{H}$ for any symmetric matrix $G$, and if $G>0$, then $\mathcal{H}=\mathcal{H}^{*}>0$. Assuming that $G=G^{*}>0$ and taking into account the relation $\mathbf{V}=\mathcal{T} \mathbf{U}$ and the boundary conditions $\left.\mathbf{V}_{1}\right|_{x_{1}=0}=\left.\mathcal{S} \mathbf{V}_{2}\right|_{x_{1}=0}$, we have

$$
-\left.\left(\mathcal{A}_{1} \mathbf{U}, \mathbf{U}\right)\right|_{x_{1}=0}=\left.\left(G \mathbf{V}_{2}, \mathbf{V}_{2}\right)\right|_{x_{1}=0} \geq\left.\delta|\mathbf{U}|_{x_{1}=0}\right|^{2},
$$

where $\delta>0$ is a constant depending on the norms of the matrices $G, \mathcal{S}$, and $\mathcal{T}$. Thus, $\mathbb{S}$ is the strictly dissipative 0 -symmetrizer.

Remark 2.3. The constants $p_{1}, p_{2}$, and $p_{3}$ are found explicitly from (2.15) through the elements of the matrix $G=\left\{g_{i j}\right\}_{i, j=1,2}$. In particular,

$$
p_{2}=-g_{22} \frac{1-b}{2(1+b)}<0
$$

$\left(g_{22}>0\right.$ since $\left.G>0\right)$. The condition $p_{2} \neq 0$ and the inequality $p_{1}^{2}-p_{2}^{2}-p_{3}^{2}>0$ imply that $\operatorname{det} \mathcal{A}_{1} \neq 0$, i.e., the boundary $x_{1}=0$ is noncharacteristic for system (2.13). This could seem strange because for system (2.11a) the boundary is characteristic, but systems (2.11a) and (2.13) are equivalent (since $\operatorname{det} P \neq 0$ ). This is, however, quite natural because we should take into account the divergent constraint (2.12). Indeed, with condition (2.12) problem (2.11) has not a so-called loss of control on derivatives in the normal direction. Namely, the $x_{1}$-derivative of the "characteristic part" $u_{3}$ of $\mathbf{U}$ is estimated from (2.12). That is, problem (2.11) being formally a hyperbolic problem with characteristic boundary has the features of noncharacteristic problems. 
Remark 2.4. The symmetrization of the multidimensional wave equation $[8,10]$ $(n \geq 3)$ with strictly dissipative boundary conditions can be formalized in terms of the construction of a strictly dissipative 0-symmetrizer as well. In particular, for the case $n=3$ the matrix $P$ has the form

$$
P=\left(\begin{array}{cccc}
p_{1} & p_{2} & p_{3} & p_{4} \\
p_{2} & p_{1} & 0 & 0 \\
p_{3} & 0 & p_{1} & 0 \\
p_{4} & 0 & 0 & p_{1}
\end{array}\right), \quad p_{i} \in \mathbb{R}, \quad p_{1}^{2}-p_{2}^{2}-p_{3}^{2}-p_{4}^{2}>0 .
$$

We refer also to [13] where such a 0-symmetrizer for the 3D wave equation is used for constructing a strictly dissipative 2-symmetrizer for relativistic gas dynamical shock waves. For simplicity, in Examples 3 and 4 below we consider the 2D case for shock waves in gas dynamics and MHD, and the structure of 2 -symmetrizers for them is based on a symmetrization of the 2D wave equation which is different from (2.13). For the 3D case for gas dynamical and MHD shock waves we refer to $[8,10,14]$ (see also further discussion in the end of section 2).

2.3. Example 2: Compressible current-vortex sheets. For tangential discontinuities (current-vortex sheets) in MHD of ideal compressible fluid, the constant coefficients linearized problem has the form of problem (2.2) (see [45]) with

$$
\begin{aligned}
& \widehat{A}_{0}^{ \pm}=\operatorname{diag}\left(\frac{1}{\hat{\rho}^{ \pm}\left(\hat{c}^{ \pm}\right)^{2}}, \hat{\rho}^{ \pm}, \hat{\rho}^{ \pm}, \hat{\rho}^{ \pm}, 1,1,1,1\right), \\
& \widehat{A}_{1}^{ \pm}=\left(\begin{array}{cccccccc}
0 & 1 & 0 & 0 & 0 & 0 & 0 & 0 \\
1 & 0 & 0 & 0 & 0 & \widehat{H}_{2}^{ \pm} & \widehat{H}_{3}^{ \pm} & 0 \\
0 & 0 & 0 & 0 & 0 & 0 & 0 & 0 \\
0 & 0 & 0 & 0 & 0 & 0 & 0 & 0 \\
0 & 0 & 0 & 0 & 0 & 0 & 0 & 0 \\
0 & \widehat{H}_{2}^{ \pm} & 0 & 0 & 0 & 0 & 0 & 0 \\
0 & \widehat{H}_{3}^{ \pm} & 0 & 0 & 0 & 0 & 0 & 0 \\
0 & 0 & 0 & 0 & 0 & 0 & 0 & 0
\end{array}\right), \\
& \widehat{A}_{2}^{ \pm}=\left(\begin{array}{cccccccc}
\frac{\hat{v}_{2}^{ \pm}}{\hat{\rho}^{ \pm}\left(\hat{c}^{ \pm}\right)^{2}} & 0 & 1 & 0 & 0 & 0 & 0 & 0 \\
0 & \hat{\rho}^{ \pm} \hat{v}_{2}^{ \pm} & 0 & 0 & -\widehat{H}_{2}^{ \pm} & 0 & 0 & 0 \\
1 & 0 & \hat{\rho}^{ \pm} \hat{v}_{2}^{ \pm} & 0 & 0 & 0 & \widehat{H}_{3}^{ \pm} & 0 \\
0 & 0 & 0 & \hat{\rho}^{ \pm} \hat{v}_{2}^{ \pm} & 0 & 0 & -\widehat{H}_{2}^{ \pm} & 0 \\
0 & -\widehat{H}_{2}^{ \pm} & 0 & 0 & \hat{v}_{2}^{ \pm} & 0 & 0 & 0 \\
0 & 0 & 0 & 0 & 0 & \hat{v}_{2}^{ \pm} & 0 & 0 \\
0 & 0 & \widehat{H}_{3} & -\widehat{H}_{2}^{ \pm} & 0 & 0 & \hat{v}_{2}^{ \pm} & 0 \\
0 & 0 & 0 & 0 & 0 & 0 & 0 & 1
\end{array}\right), \\
& \widehat{A}_{3}^{ \pm}=\left(\begin{array}{cccccccc}
\frac{\hat{v}_{3}^{ \pm}}{\hat{\rho}^{ \pm}\left(\hat{c}^{ \pm}\right)^{2}} & 0 & 0 & 1 & 0 & 0 & 0 & 0 \\
0 & \hat{\rho}^{ \pm} \hat{v}_{3}^{ \pm} & 0 & 0 & -\widehat{H}_{3}^{ \pm} & 0 & 0 & 0 \\
0 & 0 & \hat{\rho}^{ \pm} \hat{v}_{3}^{ \pm} & 0 & 0 & -\widehat{H}_{3}^{ \pm} & 0 & 0 \\
1 & 0 & 0 & \hat{\rho}^{ \pm} \hat{v}_{3}^{ \pm} & 0 & \widehat{H}_{2}^{ \pm} & 0 & 0 \\
0 & -\widehat{H}_{3}^{ \pm} & 0 & 0 & \hat{v}_{3}^{ \pm} & 0 & 0 & 0 \\
0 & 0 & -\widehat{H}_{3}^{ \pm} & \widehat{H}_{2}^{ \pm} & 0 & \hat{v}_{3}^{ \pm} & 0 & 0 \\
0 & 0 & 0 & 0 & 0 & 0 & \hat{v}_{3}^{ \pm} & 0 \\
0 & 0 & 0 & 0 & 0 & 0 & 0 & 1
\end{array}\right),
\end{aligned}
$$


$\mathbf{U}=(p, \mathbf{v}, \mathbf{H}, S), \widehat{\mathbf{U}}^{ \pm}=\left(\hat{p}^{ \pm}, 0, \hat{v}_{2}^{ \pm}, \hat{v}_{3}^{ \pm}, 0, \widehat{H}_{2}^{ \pm}, \widehat{H}_{3}^{ \pm}, \widehat{S}^{ \pm}\right)$, and the boundary conditions

$$
\begin{gathered}
f_{t}=v_{1}^{ \pm}-\hat{v}_{2}^{ \pm} f_{x_{2}}-\hat{v}_{3}^{ \pm} f_{x_{3}}, \quad[q]=0, \\
H_{1}^{ \pm}=\widehat{H}_{2}^{ \pm} f_{x_{2}}+\widehat{H}_{3}^{ \pm} f_{x_{3}}
\end{gathered}
$$

at $x_{1}=0$. Here $q=p+\left(\widehat{\mathbf{H}}^{ \pm}, \mathbf{H}\right)$ for $\mathbf{x} \in \mathbb{R}_{ \pm}^{3}, \widehat{\mathbf{H}}^{ \pm}=\left(0, \widehat{H}_{2}^{ \pm}, \widehat{H}_{3}^{ \pm}\right), \hat{p}^{ \pm}=p\left(\hat{\rho}^{ \pm}, \widehat{S}^{ \pm}\right)$, $\left(\hat{c}^{ \pm}\right)^{2}=p_{\rho}\left(\hat{\rho}^{ \pm}, \widehat{S}^{ \pm}\right)>0$, and $p=p(\rho, S)$ is the state equation of gas, $\hat{\rho}^{ \pm}>0$ is the unperturbed density for $x_{1} \gtrless 0$, etc. (see [45]). As in [45], without loss of generality we suppose that $\sigma=0$ (see $(2.1)$ ), i.e., $\widehat{A}_{\nu}^{ \pm}=\widehat{A}_{1}^{ \pm}$and consider the homogenous problem $\left(\mathbf{f}^{ \pm}=0\right.$ and $\left.\mathbf{g}=0\right)$.

Remark 2.5. Since $\operatorname{det} \widehat{A}_{1}^{ \pm}=0$, the boundary $x_{1}=0$ is characteristic, i.e., current-vortex sheet is a characteristic discontinuity. For shock waves the correct number of boundary conditions is guaranteed by the Lax conditions (1.20). For the general case, the number of boundary conditions should be equal to

$$
1+\sum_{ \pm} \# \text { positive eigenvalues of } \pm A_{\nu}^{ \pm} \text {counting multiplicity. }
$$

That is, for current-vortex sheets the correct number of boundary conditions is three. At first sight, problem (2.2a), (2.16) is overdetermined. On the other hand, one can show that for the original nonlinear problem the boundary conditions $\left(\mathbf{H}^{ \pm}, \mathbf{N}\right)=0$ (see [45]) can be regarded as the restrictions only on the initial data. This was shown in [46] for the case of incompressible MHD, but this proposition can be easily proved for compressible current-vortex sheets as well. Of course, this fact can be analogously (and easier) proved for the linear problem. That is, for problem (2.2a), (2.16) the boundary conditions (2.16b) are just the restrictions on the initial data.

Unlike, for example, MHD shock waves or Alfvén discontinuities [13, 44], for current-vortex sheets the Lopatinski determinant can be explicitly written out. At the same time, it is reduced to an algebraic equation of the tenth degree depending on seven dimensionless parameters and one more inner parameter determining the wave vector (see [45]). Moreover, the squaring was applied under the reduction of the Lopatinski determinant to this algebraic equation and, therefore, it can introduce spurious roots. For all these reasons both the analytical analysis and the full numerical study of the Lopatinski determinant are unacceptable for finding the Lopatinski condition. Although, one can analytically show that the uniform Lopatinski condition is never satisfied for problem (2.2a), (2.16), i.e., planar current-vortex sheets can be either violently unstable or weakly (neutrally) stable (see [45]). The alternative energy method suggested in [45] has first enabled one to find sufficient conditions for their weak stability.

The method in [45] can be now described in terms of the notation of dissipative symmetrizer. In fact, in [45] the dissipative 0-symmetrizer $\mathbb{S}=\left\{P^{+}, P^{-}, \mathbf{R}^{+}, \mathbf{R}^{-}\right\}$ was suggested for problem (2.2a), (2.16), where $P^{ \pm}=P\left(\widehat{\mathbf{U}}^{ \pm}\right), \mathbf{R}^{ \pm}=\lambda\left(\widehat{\mathbf{U}}^{ \pm}\right) \mathbf{R}\left(\widehat{\mathbf{U}}^{ \pm}\right)$,

$$
P=\left(\begin{array}{cccccccc}
1 & \frac{\lambda H_{1}}{\rho c^{2}} & \frac{\lambda H_{2}}{\rho c^{2}} & \frac{\lambda H_{3}}{\rho c^{2}} & 0 & 0 & 0 & 0 \\
\lambda H_{1} \rho & 1 & 0 & 0 & -\rho \lambda & 0 & 0 & 0 \\
\lambda H_{2} \rho & 0 & 1 & 0 & 0 & -\rho \lambda & 0 & 0 \\
\lambda H_{3} \rho & 0 & 0 & 1 & 0 & 0 & -\rho \lambda & 0 \\
0 & -\lambda & 0 & 0 & 1 & 0 & 0 & 0 \\
0 & 0 & -\lambda & 0 & 0 & 1 & 0 & 0 \\
0 & 0 & 0 & -\lambda & 0 & 0 & 1 & 0 \\
0 & 0 & 0 & 0 & 0 & 0 & 0 & 1
\end{array}\right), \quad \mathbf{R}=-\left(\begin{array}{c}
1 \\
0 \\
0 \\
0 \\
H_{1} \\
H_{2} \\
H_{3} \\
0
\end{array}\right)
$$


$\lambda=\lambda(\mathbf{U})$ is a function, and the constants $\lambda^{ \pm}=\lambda\left(\widehat{\mathbf{U}}^{ \pm}\right)$are chosen below. Actually, the application of $\{P, \mathbf{R}\}$ to the original nonlinear MHD system gives a new symmetric form [45] of the MHD equations with the hyperbolicity condition, $\mathcal{A}_{0}(\mathbf{U})=P A_{0}>0$,

$$
\rho \lambda^{2}<\frac{1}{1+c_{\mathrm{A}}^{2} / c^{2}},
$$

where $c_{\mathrm{A}}=|\mathbf{H}| / \sqrt{\rho}$ (see [45]).

Note that for current sheets, i.e., for the case when $[\hat{\mathbf{v}}]=0\left(\hat{\mathbf{v}}^{ \pm}=\left(0, \hat{v}_{2}^{ \pm}, \hat{v}_{3}^{ \pm}\right)\right)$the boundary conditions (2.16) are dissipative:

$$
\left.\left[\left(\widehat{A}_{1} \mathbf{U}, \mathbf{U}\right)\right]\right|_{x_{1}=0}=2 q^{+}\left[v_{1}\right]=2 q^{+}\left(\left[\hat{\mathbf{v}}^{\prime}\right], \nabla_{\mathbf{x}^{\prime}} f\right)=0,
$$

where $\hat{\mathbf{v}}^{\prime \pm}=\left(\hat{v}_{2}^{ \pm}, \hat{v}_{3}^{ \pm}\right)$. That is, for current sheets one has the identical 0-symmetrizer $\mathbb{S}=\{I, 0, I, 0\}\left(\lambda^{ \pm}=0\right)$. Suppose now that $[\hat{\mathbf{v}}] \neq 0$ and $\widehat{\mathbf{H}}^{+} \times \widehat{\mathbf{H}}^{-} \neq 0$ (for the particular case $\widehat{\mathbf{H}}^{+} \times \widehat{\mathbf{H}}^{-}=0$ we refer to [45]). The matrices $\widehat{\mathcal{A}}_{1}^{ \pm}$(cf. (2.5)) have the form

$$
\widehat{\mathcal{A}}_{1}^{ \pm}=\left(\begin{array}{cccccccc}
0 & 1 & 0 & 0 & -\lambda^{ \pm} & 0 & 0 & 0 \\
1 & 0 & 0 & 0 & 0 & \widehat{H}_{2}^{ \pm} & \widehat{H}_{3}^{ \pm} & 0 \\
0 & 0 & 0 & 0 & 0 & 0 & 0 & 0 \\
0 & 0 & 0 & 0 & 0 & 0 & 0 & 0 \\
-\lambda^{ \pm} & 0 & 0 & 0 & 0 & -\lambda^{ \pm} \widehat{H}_{2}^{ \pm} & -\lambda^{ \pm} \widehat{H}_{3}^{ \pm} & 0 \\
0 & \widehat{H}_{2}^{ \pm} & 0 & 0 & -\lambda^{ \pm} \widehat{H}_{2}^{ \pm} & 0 & 0 & 0 \\
0 & \widehat{H}_{3}^{ \pm} & 0 & 0 & -\lambda^{ \pm} \widehat{H}_{3}^{ \pm} & 0 & 0 & 0 \\
0 & 0 & 0 & 0 & 0 & 0 & 0 & 0
\end{array}\right)
$$

Then, by virtue of (2.16),

$$
\left.\left[\left(\widehat{\mathcal{A}}_{1} \mathbf{U}, \mathbf{U}\right)\right]\right|_{x_{1}=0}=2 q^{+}\left[v_{1}-\lambda H_{1}\right]=2 q^{+}\left(\left[\hat{\mathbf{v}}^{\prime}-\lambda \widehat{\mathbf{H}}^{\prime}\right], \nabla_{\mathrm{x}^{\prime}} f\right),
$$

where $\widehat{\mathbf{H}}^{\prime \pm}=\left(\widehat{H}_{2}^{ \pm}, \widehat{H}_{3}^{ \pm}\right)$.

The constants $\lambda^{ \pm}$are chosen so that $\left[\hat{\mathbf{v}}^{\prime}-\lambda \widehat{\mathbf{H}}^{\prime}\right]=0$ :

$$
\lambda^{ \pm}=-\frac{|[\hat{\mathbf{v}}]| \sin \varphi^{\mp}}{\left|\widehat{\mathbf{H}}^{ \pm}\right| \sin \left(\varphi^{+}-\varphi^{-}\right)}, \quad \cos \varphi^{ \pm}=\frac{\left([\hat{\mathbf{v}}], \widehat{\mathbf{H}}^{ \pm}\right)}{|[\hat{\mathbf{v}}]|\left|\widehat{\mathbf{H}}^{ \pm}\right|} .
$$

For such $\lambda^{ \pm}$the boundary conditions for system (2.5) are dissipative and, therefore, $\mathbb{S}$ is the dissipative (but not strictly dissipative) 0 -symmetrizer, provided that $\widehat{\mathcal{A}}_{0}^{ \pm}=$ $\mathcal{A}_{0}\left(\widehat{\mathbf{U}}^{ \pm}\right)>0$. In view of $(2.17)$, the last conditions for the chosen $\lambda^{ \pm}$read:

$$
|[\hat{\mathbf{v}}]|<\left|\sin \left(\varphi^{+}-\varphi^{-}\right)\right| \min \left\{\frac{\gamma^{+}}{\left|\sin \varphi^{-}\right|}, \frac{\gamma^{-}}{\left|\sin \varphi^{+}\right|}\right\}
$$

where $\gamma^{ \pm}=\hat{c}^{ \pm} \hat{c}_{\mathrm{A}}^{ \pm} /\left(\left(\hat{c}^{ \pm}\right)^{2}+\left(\hat{c}_{\mathrm{A}}^{ \pm}\right)^{2}\right)^{1 / 2}$. Inequality (2.18) represents the sufficient condition for the neutral stability of compressible current-vortex sheets. This condition is of importance for various astrophysical applications such as, for example, the heliopause model [4]. As was shown in [45, 46], in the incompressibility limit inequality (2.18) describes exactly half of the parameter domain of neutral stability. 
2.4. Example 3: Gas dynamical shock waves. The system of gas dynamics is an unconstrained hyperbolic system, and gas dynamical shock waves are known to be 1-shocks. Therefore, while constructing a strictly dissipative symmetrizer one can suppose that $P^{-}=\gamma I$ (see Remark 2.1) and, clearly, $\mathbf{R}_{j, \alpha}^{ \pm}=0$. Keeping in mind the observation about $P^{-}$, without loss of generality the perturbation ahead the planar 1-shock can be assumed to be equal to zero: $\mathbf{U}=0$ for $x_{1}<0$. That is, for gas dynamical shocks the constant coefficients linearized problem (see (2.2)) is formulated in the half-space $\mathbb{R}_{+}^{n}$ and the strictly dissipative $p$-symmetrizer for it is just a matrix: $\mathbb{S}=P^{+}$.

For the $2 \mathrm{D}$ case $(n=2)$ and in dimensionless values the constant coefficients linearized problem for gas dynamical shock waves has the form of (2.2) with $\left.\mathbf{U}\right|_{x_{1}<0}=$ 0 and $\widehat{A}_{0}^{+}=\operatorname{diag}\left(1, M^{2}, M^{2}, 1\right)$,

$$
\widehat{A}_{\nu}^{+}=\widehat{A}_{1}^{+}=\left(\begin{array}{cccc}
1 & 1 & 0 & 0 \\
1 & M^{2} & 0 & 0 \\
0 & 0 & M^{2} & 0 \\
0 & 0 & 0 & 1
\end{array}\right), \quad \widehat{A}_{2}^{+}=\left(\begin{array}{cccc}
0 & 0 & 1 & 0 \\
0 & 0 & 0 & 0 \\
1 & 0 & 0 & 0 \\
0 & 0 & 0 & 0
\end{array}\right)
$$

(without loss of generality $\boldsymbol{\sigma}=0, \mathbf{f}^{ \pm}=0$, and $\mathbf{g}=0$ ). Here $M=\hat{v}_{1}^{+} / \hat{c}^{+}$is the Mach number behind the shock (in view of the Lax shock conditions, $M<1$ ), $\widehat{\mathbf{U}}^{ \pm}=$ $\left(\hat{p}^{ \pm}, \hat{v}_{1}^{ \pm}, 0, \widehat{S}^{ \pm}\right),\left(\hat{c}^{ \pm}\right)^{2}=\left(\rho^{2} E_{\rho}\right)_{\rho}\left(\hat{\rho}^{ \pm}, \widehat{S}^{ \pm}\right)>0$, and $E=E(\rho, S)$ is the state equation of gas, etc. (see, e.g., [13]). The vector $\mathbf{U}=\left(p, v_{1}, v_{2}, S\right)$ is the vector of perturbations in dimensionless values [13] and $\widehat{A}_{\alpha}^{+}=D\left(\widehat{\mathbf{U}}^{+}\right)^{*} A_{\alpha}\left(\widehat{\mathbf{U}}^{+}\right) D\left(\widehat{\mathbf{U}}^{+}\right)$, where $D\left(\widehat{\mathbf{U}}^{+}\right)$is a diagonal matrix reducing system (2.2a) $\left.\right|_{x_{1}>0}$ to a dimensionless form.

The boundary conditions in a dimensionless form and after eliminating the function $f\left(t, x_{2}\right)$ read [13]:

$$
v_{1}+b_{1} p=0, \quad\left(v_{2}\right)_{t}=b_{2} p_{x_{2}}, \quad S=b_{3} p,
$$

where

$$
b_{1}=\frac{a+1}{2 M^{2}}, \quad b_{2}=\frac{(a-1) R}{2 M^{2}}, \quad b_{3}=1-\frac{a}{M^{2}}, \quad a=\frac{h-R+1}{h / M^{2}-R+1},
$$

$R=\hat{\rho}^{+} / \hat{\rho}^{-}, h=\left(2 E_{S} /\left(\rho E_{\rho S}\right)\right)\left(\hat{\rho}^{+}, \widehat{S}^{+}\right)$. As is known (see [13, 34] and references therein), the boundary conditions (2.19) satisfy the uniform Lopatinski condition, i.e., planar gas dynamical shock waves are uniformly stable if and only if

$$
\frac{M^{2}(R+1)-1}{M^{2}(R-1)+1}<a<1 .
$$

The energy method enabling one to deduce an a priori estimate with no loss of derivatives for (2.2a), (2.19), provided that the uniform Lopatinski condition (2.20) holds, was suggested by Blokhin [6]. We now formalize this method by writing out a strictly dissipative 2-symmetrizer for this problem (for the 3D case we refer to [13] and references therein). The construction of this symmetrizer is based on a certain symmetrization of the wave equation for the pressure perturbation $p$ implied by the acoustics system:

$$
\tilde{\partial}_{t}^{2} p-\tilde{\partial}_{1}^{2} p-\tilde{\partial}_{2}^{2} p=0,
$$


where

$$
\tilde{\partial}_{t}=\frac{M}{b^{2}} \partial_{t}, \quad \tilde{\partial}_{1}=\partial_{1}-\frac{M^{2}}{b^{2}} \partial_{t}, \quad \tilde{\partial}_{2}=\frac{1}{b} \partial_{2}, \quad b=\sqrt{1-M^{2}} \in \mathbb{R}_{+} .
$$

Using the boundary conditions (2.19) and the acoustics system itself, one can obtain the following boundary condition for $(2.21)$ :

$$
M^{2}\left(1+b_{1}\right) p_{t t}-b^{2} p_{t x_{1}}+M^{2} b_{2} p_{x_{2} x_{2}}=0, \quad x_{1}=0 .
$$

Problem (2.21), (2.22) is "symmetrized" as follows (see [13] for details):

$$
\begin{gathered}
B_{0} \mathbf{Y}_{t}+B_{1} \mathbf{Y}_{x_{1}}+B_{2} \mathbf{Y}_{x_{2}}=0 \text { for } x_{1}>0 \\
\mathcal{M} \mathbf{Y}=0 \quad \text { for } x_{1}=0
\end{gathered}
$$

where

$$
\begin{aligned}
& \mathbf{Y}=\left(\begin{array}{c}
\mathbf{Y}_{1} \\
\mathbf{Y}_{2} \\
\mathbf{Y}_{3}
\end{array}\right), \quad \mathbf{Y}_{1}=\tilde{\partial}_{t}\left(\begin{array}{c}
\tilde{\partial}_{t} p \\
\tilde{\partial}_{1} p \\
\tilde{\partial}_{2} p
\end{array}\right), \quad \mathbf{Y}_{i}=\tilde{\partial}_{i-1}\left(\begin{array}{c}
\tilde{\partial}_{t} p \\
\tilde{\partial}_{1} p \\
\tilde{\partial}_{2} p
\end{array}\right), \quad i=2,3 \\
& B_{0}=\frac{M}{b^{2}} \mathcal{T}^{*}\left\{\left(\begin{array}{cc}
1 & -M \\
-M & 1
\end{array}\right) \otimes \mathcal{H}\right\} \mathcal{T}, \quad B_{1}=\mathcal{T}^{*}\left\{\left(\begin{array}{ll}
0 & 1 \\
1 & 0
\end{array}\right) \otimes \mathcal{H}\right\} \mathcal{T}, \\
& B_{2}=\frac{1}{b} \mathcal{T}^{*}\left\{\left(\begin{array}{cc}
1 & 0 \\
0 & -1
\end{array}\right) \otimes \mathcal{H}\right\} \mathcal{T}, \quad \mathcal{T}=\frac{1}{\sqrt{2}}\left(\begin{array}{ccc}
1 & 0 & -1 \\
0 & -1 & 0 \\
0 & -1 & 0 \\
1 & 0 & 1
\end{array}\right) \otimes I_{3} \\
& \mathcal{H}=\left(\begin{array}{cc}
P_{1}-P_{3} & -P_{2}-P_{4} \\
-P_{2}+P_{4} & P_{1}+P_{3}
\end{array}\right), \quad \mathcal{M}=\left(\begin{array}{lll}
\mathcal{M}_{1} & \mathcal{M}_{2} & \mathcal{M}_{3}
\end{array}\right), \\
& \mathcal{M}_{1}=\left(\begin{array}{lll}
1 & 2 & 0 \\
0 & 0 & 0 \\
0 & 1 & 0
\end{array}\right), \quad \mathcal{M}_{2}=\left(\begin{array}{ccc}
-2 & -1 & 0 \\
0 & 0 & -1 \\
0 & -M b_{1} & 0
\end{array}\right), \quad \mathcal{M}_{3}=\left(\begin{array}{ccc}
0 & 0 & -1 \\
0 & 1 & 0 \\
0 & 0 & -b_{0}
\end{array}\right) \text {, }
\end{aligned}
$$

$P_{k}(k=\overline{1,3})$ are arbitrary symmetric matrices of order $3, P_{4}$ is an arbitrary antisymmetric matrix of order 3 , and $b_{0}=M b_{1}+\left(M^{3} b_{2} / b^{2}\right)$.

Referring for detailed arguments to [13], one gets (cf. (2.14))

$$
-\left.\left(B_{1} \mathbf{Y}, \mathbf{Y}\right)\right|_{x_{1}=0}=-\left.\left(\left\{\mathcal{S}^{*} \mathcal{H}+\mathcal{H S}\right\} \mathbf{V}_{2}, \mathbf{V}_{2}\right)\right|_{x_{1}=0}
$$

where

$$
\begin{gathered}
\mathbf{V}=\mathcal{T} \mathbf{Y}=\left(\begin{array}{c}
\mathbf{V}_{1} \\
\mathbf{V}_{2}
\end{array}\right),\left.\quad \mathbf{V}_{1}\right|_{x_{1}=0}=\left.\mathcal{S} \mathbf{V}_{2}\right|_{x_{1}=0}, \quad \mathcal{S}=\left(\begin{array}{cc}
\mathcal{S}_{1} & -\mathcal{S}_{2} \\
I_{3} & 0
\end{array}\right) \\
\mathcal{S}_{1}=2\left(\mathcal{M}_{1}-\mathcal{M}_{3}\right)^{-1} \mathcal{M}_{2}, \quad \mathcal{S}_{2}=\left(\mathcal{M}_{1}-\mathcal{M}_{3}\right)^{-1}\left(\mathcal{M}_{1}+\mathcal{M}_{3}\right) .
\end{gathered}
$$

One can show that all the eigenvalues of the matrix $\mathcal{S}$ lie strictly in the left half-plane $\left(\Re \lambda_{i}(\mathcal{S})<0, i=\overline{1,6}\right)$ if and only if the uniform Lopatinski condition $(2.20)$ holds. In this case the Lyapunov matrix equation in the form of (2.15) has the unique solution 
$\mathcal{H}=\mathcal{H}^{*}>0$ for any symmetric and positive definite matrix $G$. Moreover, since $\mathcal{H}>0$, the matrix $B_{0}>0$. The assumption $G>0$ yields

$$
-\left.\left(B_{1} \mathbf{Y}, \mathbf{Y}\right)\right|_{x_{1}=0}=\left.\left(G \mathbf{V}_{2}, \mathbf{V}_{2}\right)\right|_{x_{1}=0} \geq C_{1}\left|\mathbf{X}^{+}\right|^{2},
$$

where

$$
\mathbf{X}=\left(p_{t t}, p_{t x_{1}}, p_{t x_{2}}, p_{x_{1} x_{1}}, p_{x_{1} x_{2}}, p_{x_{2} x_{2}}\right), \quad \mathbf{X}^{+}=\left.\mathbf{X}\right|_{x_{1}=0}, \quad \mathbf{Y}=\mathcal{K} \mathbf{X}
$$

$\mathcal{K}$ is a $9 \times 6$ matrix which can be explicitly written out, and $C_{1}=C_{1}(G)>0$ is a constant depending on the norm of the matrix $G$ (as well as on the norms of the matrices $\mathcal{S}, \mathcal{T}$, and $\mathcal{K}$ ).

In fact, we have constructed the strictly dissipative 1-symmetrizer for the subproblem, $(2.21),(2.22)$, for the vector $\left(p_{t}, p_{x_{1}}, p_{x_{2}}\right)$. We are now ready to describe the strictly dissipative 2-symmetrizer for the whole problem, (2.2a), (2.19). It has the form

$$
\mathbb{S}=P^{+}=I_{24}+\mathcal{N}^{*} \mathcal{K}^{*} B_{0} \mathcal{K} \mathcal{N}\left(I_{6} \otimes\left(\widehat{A}_{0}^{+}\right)^{-1}\right)
$$

where $\mathcal{N}$ is the projector of $\mathbf{W}=\mathbf{W}_{2}=\left(\mathbf{U}_{t t}, \mathbf{U}_{t x_{1}}, \ldots, \mathbf{U}_{x_{2} x_{2}}\right)$ on $\mathbf{X}$, i.e., $\mathbf{X}=\mathcal{N} \mathbf{W}$.

It is clear that $\widehat{\mathcal{A}}_{0}^{+}=P^{+}\left(I_{6} \otimes \widehat{A}_{0}^{+}\right)>0$. Indeed, $\left(\widehat{\mathcal{A}}_{0}^{+} \mathbf{W}, \mathbf{W}\right)=\left(\left(I_{6} \otimes \widehat{A}_{0}^{+}\right) \mathbf{W}, \mathbf{W}\right)+$ $\left(B_{0} \mathbf{Y}, \mathbf{Y}\right)>0$ for all $\mathbf{W} \neq 0$. Concerning condition (2.8), one has

$$
-\left.\left(\widehat{\mathcal{A}}_{1}^{+} \mathbf{W}, \mathbf{W}\right)\right|_{x_{1}=0}=-\left.\left(\left(I_{6} \otimes \widehat{A}_{1}^{+}\right) \mathbf{W}, \mathbf{W}\right)\right|_{x_{1}=0}-\left.\left(B_{1} \mathbf{Y}, \mathbf{Y}\right)\right|_{x_{1}=0}
$$

Using the boundary conditions (2.19) and the acoustics system it is not difficult to show that $\left.\mathbf{W}\right|_{x_{1}=0}=\left.B \mathbf{X}\right|_{x_{1}=0}$, where $B$ is a $24 \times 6$ matrix with elements depending on the coefficients of (2.19) and the matrices $\widehat{A}_{\alpha}^{+}$. Therefore, there exists a positive constant $C_{2}$ such that

$$
-\left.\left(\left(I_{6} \otimes \widehat{A}_{1}^{+}\right) \mathbf{W}, \mathbf{W}\right)\right|_{x_{1}=0} \geq-C_{2}\left|\mathbf{X}^{+}\right|^{2} .
$$

Analogous arguments show that $\left|\mathbf{X}^{+}\right|^{2} \geq C_{3}\left|\mathbf{W}^{+}\right|^{2}$ with a constant $C_{3}>0$. On the other hand, by an appropriate choice of the matrix $G$ (i.e., the choice of matrices $P_{i}$ ) one can achieve that $C_{1}-C_{2}>0$. Hence,

$$
-\left.\left(\widehat{\mathcal{A}}_{1}^{+} \mathbf{W}, \mathbf{W}\right)\right|_{x_{1}=0} \geq\left(C_{1}-C_{2}\right)\left|\mathbf{X}^{+}\right|^{2} \geq \delta\left|\mathbf{W}^{+}\right|^{2},
$$

where $\delta=\left(C_{1}-C_{2}\right) C_{3}>0$. Thus, $\mathbb{S}$ is indeed the strictly dissipative 2 -symmetrizer.

2.5. Example 4: Fast MHD shock waves. As gas dynamical shock waves, fast MHD shock waves are also 1-shocks (see, e.g., [13]). That is, the constant coefficient linearized problem for fast MHD shocks in 2D (for 3D see [14]) and in dimensionless values has the form of the problem for gas dynamical shock waves formulated above, with $\widehat{A}_{0}^{+}=\operatorname{diag}\left(1, M^{2}, M^{2}, 1,1,1\right)$,

$$
\widehat{A}_{1}^{+}=\left(\begin{array}{cccccc}
1 & 1 & 0 & 0 & 0 & 0 \\
1 & M^{2} & 0 & 0 & h_{2} & 0 \\
0 & 0 & M^{2} & 0 & -h_{1} & 0 \\
0 & 0 & 0 & 1 & 0 & 0 \\
0 & h_{2} & -h_{1} & 0 & 1 & 0 \\
0 & 0 & 0 & 0 & 0 & 1
\end{array}\right), \quad \widehat{A}_{2}^{+}=\left(\begin{array}{cccccc}
0 & 0 & 1 & 0 & 0 & 0 \\
0 & 0 & 0 & -h_{2} & 0 & 0 \\
1 & 0 & 0 & h_{1} & 0 & 0 \\
0 & -h_{2} & h_{1} & 0 & 0 & 0 \\
0 & 0 & 0 & 0 & 0 & 0 \\
0 & 0 & 0 & 0 & 0 & 0
\end{array}\right),
$$


$\widehat{\mathbf{U}}^{ \pm}=\left(\hat{p}^{ \pm}, \hat{v}_{1}^{ \pm}, \hat{v}_{2}^{ \pm}, \widehat{H}_{1}^{ \pm}, \widehat{H}_{2}^{ \pm}, \widehat{S}^{ \pm}\right), \hat{v}_{2}^{+}=0, h_{i}=\widehat{H}_{i}^{+} /(\hat{c} \sqrt{\hat{\rho}}), \mathbf{U}=\left(p, v_{1}, v_{2}, H_{1}, H_{2}, S\right)$, etc. (see $[12,13])$. The boundary conditions read

$$
\begin{gathered}
v_{1}+b_{1} p=q_{1} f_{x_{2}}, \quad f_{t}=b_{2} p+q_{2} f_{x_{2}}, \quad v_{2}=b_{3} f_{x_{2}}+q_{3} p, \\
H_{2}=h_{2} b_{0} f_{t}-h_{2} v_{1}+h_{1} v_{2}, \quad H_{1}=h_{2} b_{0} f_{x_{2}}, \quad S=b_{4} p+q_{4} f_{x_{2}},
\end{gathered}
$$

where the coefficients $b_{i}$ are explicitly written out in $[12,13]$ for the case of a polytropic gas and a weak magnetic field, $q=\sqrt{h_{1}^{2}+h_{2}^{2}} \ll 1$ (for the general case, $q \in(0,+\infty)$, see [44]), the coefficients $q_{i}=O\left(q^{2}\right)$ for $q \ll 1$, and $b_{0}=1-\left(\widehat{H}_{2}^{-} / \widehat{H}_{2}^{+}\right) \in(0,1)$ (for parallel shocks, $\left.\widehat{H}_{2}^{ \pm}=0, b_{0}:=0\right)$.

The energy method suggested in [12] is based on the fact that the magnetoacoustics system implies the wave equation with an additional "magnetic" term:

$$
\tilde{\partial}_{t}^{2} p-\tilde{\partial}_{1}^{2} p-\tilde{\partial}_{2}^{2} p+\frac{q}{b^{2}} \triangle Q=0
$$

(notations are the same as in $(2.21))$, where $Q=(\mathbf{b}, \mathbf{H}), \mathbf{H}=\left(H_{1}, H_{2}\right)$, and $\mathbf{b}=$ $\left(-h_{1} / q, h_{2} / q\right)(|\mathbf{b}|=1)$. Then, the counterpart of system (2.23) reads

$$
B_{0} \mathbf{Y}_{t}+B_{1} \mathbf{Y}_{x_{1}}+B_{2} \mathbf{Y}_{x_{2}}+\frac{q}{b^{2}}\left(\begin{array}{c}
P_{1} \\
P_{2} \\
P_{3}
\end{array}\right) \triangle \mathbf{Q}=0 \text { for } x_{1}>0,
$$

where $\mathbf{Q}=\left(\tilde{\partial}_{t} Q, \tilde{\partial}_{1} Q, \tilde{\partial}_{2} Q\right)$. Moreover, for the function $p$ we can obtain a counterpart of the boundary condition $(2.22)$ (see $[12,14]$ ) which implies $(2.24)$ with the matrices $\mathcal{M}_{i}$ being slightly different from the corresponding matrices in gas dynamics for the case $q \ll 1$ (the norms of the differences are of order $O\left(q^{2}\right)$ ).

The crucial role in deducing the a priori estimate [12] for $(2.2 \mathrm{a}),(2.25)$ is played by the important fact that the term

$$
\left(\mathbf{Y},\left(\begin{array}{c}
P_{1} \\
P_{2} \\
P_{3}
\end{array}\right) \triangle \mathbf{Q}\right)=\sum_{i=1}^{3}\left(\mathbf{Y}_{i}, P_{i} \triangle \mathbf{Q}\right)
$$

can be represented in a divergent form,

$$
\sum_{i=1}^{3}\left(\mathbf{Y}_{i}, P_{i} \triangle \mathbf{Q}\right)=\left(R_{0} \mathbf{W}, \mathbf{W}\right)_{t}+\left(R_{1} \mathbf{W}, \mathbf{W}\right)_{x_{1}}+\left(R_{2} \mathbf{W}, \mathbf{W}\right)_{x_{2}}
$$

where $\mathbf{W}=\mathbf{W}_{2}$, and the quadratic forms $\left(R_{\alpha} \mathbf{W}, \mathbf{W}\right)$ are explicitly written out in $[12,13]$ (if necessary, the symmetric matrices $R_{\alpha}$ of order 36 can be written out as well). While obtaining representation (2.28) the divergent constraint $\operatorname{div} \mathbf{H}=0$ and the magnetoacoustics system itself were essentially used.

The strictly dissipative 2-symmetrizer for fast MHD shock waves has the form

$$
\mathbb{S}=\left\{P^{+},\left\{\mathbf{R}_{1, \alpha}^{+}\right\}|\alpha|=2\right\}
$$

with

$$
P^{+}=I_{36}+\mathcal{N}^{*} \mathcal{K}^{*} B_{0} \mathcal{K} \mathcal{N}\left(I_{6} \otimes\left(\widehat{A}_{0}^{+}\right)^{-1}\right)+P_{0}^{+}, \quad\left\{\mathbf{R}_{1, \alpha}^{+}\right\}_{|\alpha|=2}=\left\{\mathbf{R}_{1}, \ldots, \mathbf{R}_{6}\right\} .
$$

Here $\mathcal{N}$ is the projector of $\mathbf{W}$ on $\mathbf{X}$, the matrices $\mathcal{K}$ and $B_{0}$ and the vector $\mathbf{X}$ are the same as for gas dynamical shocks, the matrix $P_{0}^{+}$and the vectors $\mathbf{R}_{i}$, which are of 
order $O(q)$ for $q \ll 1$, can be explicitly written out by analyzing representation (2.28). Moreover,

$$
\begin{gathered}
\left(\widehat{\mathcal{A}}_{0}^{+} \mathbf{W}, \mathbf{W}\right)=\left(\left(I_{6} \otimes \widehat{A}_{0}^{+}\right) \mathbf{W}, \mathbf{W}\right)+\left(B_{0} \mathbf{Y}, \mathbf{Y}\right)+\frac{2 q}{b^{2}}\left(R_{0} \mathbf{W}, \mathbf{W}\right) \\
-\left.\left(\widehat{\mathcal{A}}_{1}^{+} \mathbf{W}, \mathbf{W}\right)\right|_{x_{1}=0}=-\left.\left(\left(I_{6} \otimes \widehat{A}_{1}^{+}+\frac{2 q}{b^{2}} R_{0}\right) \mathbf{W}, \mathbf{W}\right)\right|_{x_{1}=0}-\left.\left(B_{1} \mathbf{Y}, \mathbf{Y}\right)\right|_{x_{1}=0}
\end{gathered}
$$

It is clear that for $\mathcal{H}>0$ and $q \ll 1$ the matrix $\widehat{\mathcal{A}}_{0}^{+}>0$. For the case of a weak magnetic field $(q \ll 1)$ and a polytropic gas, one can show that all the eigenvalues of the matrix $\mathcal{S}$ lie strictly in the left half-plane. That is, $-\left.\left(\widehat{\mathcal{A}}_{1}^{+} \mathbf{W}, \mathbf{W}\right)\right|_{x_{1}=0} \geq \delta\left|\mathbf{W}^{+}\right|^{2}$, where the constant $\delta>0$ for $q \ll 1$ (see $[12,13]$ for more details). Thus, $\mathbb{S}$ is indeed the strictly dissipative 2-symmetrizer of (2.2a), (2.25) for the case of a weak magnetic field and a polytropic gas. Concerning the case of a general equation of state, the same is true if we require the fulfillment of (2.20) that is in fact the uniform Lopatinski condition for fast MHD shocks for $q \ll 1$.

Remark 2.6. In principle, for the general case, $q \in(0,+\infty)$, we can try to find the conditions for $\widehat{\mathbf{U}}^{ \pm}$guaranteeing the fulfillment of the requirements $\widehat{\mathcal{A}}_{0}^{+}>0$ and $-B^{*} \widehat{\mathcal{A}}_{1}^{+} B>0$, where $\mathbf{W}^{+}=B \mathbf{X}^{+}$. Then, $\mathbb{S}$ is the strictly dissipative 2 -symmetrizer, provided that these conditions hold. However, rather cumbersome calculations should be unfortunately performed to find the mentioned conditions. At the same time, if they are possible to be found at least numerically, for fixed parameters $\widehat{\mathbf{U}}^{ \pm}$, then it is interesting to compare them with the uniform stability domain for fast MHD shock waves. This domain was found in [44] by numerical testing of the uniform Lopatinski condition with the help of an algorithm suggested for 1-shocks.

2.6. Further examples. For gas dynamical shock waves we have presented a strictly dissipative 2 -symmetrizer for the $2 \mathrm{D}$ case. For the $3 \mathrm{D}$ case the structure of the 2-symmetrizer is different, but the process of construction of this symmetrizer is also based on using a symmetrization of the wave equation. The same structure has the strictly dissipative 2-symmetrizer for shock waves in relativistic gas dynamics (see [13]) and in nonrelativistic radiation hydrodynamics [3]. For shock waves in relativistic radiation hydrodynamics [15] the structure of the strictly dissipative 2-symmetrizer is a little bit more complicated; however, it refers to a symmetrization of the wave equation as well.

It is interesting to note that, for instance, the system of Landau's equations of superfluid [29] is a constrained hyperbolic system, but the relations $\nabla \times \mathbf{v}_{\mathbf{s}}=0$ are not used under the construction of the strictly dissipative 2-symmetrizer for shock waves in this model (see [11] and references therein), i.e., $\mathbf{R}_{j, \alpha}^{ \pm}=0$. Unfortunately, there is not a general method to construct a (strictly) dissipative $p$-symmetrizer. For most concrete examples $p=2$ and they are based on using different symmetrizations of the wave equation. So, Definition 2.1 was given for $p \geq 0$, but we will privately suppose that $p=0$ or $p=1$ or $p=2$ (actually, we do not know examples with $p \geq 3$ ). Moreover, for the physical cases $n=2$ and $n=3$ (2D and 3D) the existence of a strictly dissipative $p$-symmetrizer with $p \geq 3$ implies a weaker local existence theorem for the nonlinear problem (in the generic case, for a symmetrizer with $p \geq[n / 2]+2$ one obtains a weaker nonlinear result, see section 4).

Observe that in [10] also a strictly dissipative 1-symmetrizer was in fact constructed for gas dynamical shock waves. But in this case the domain $D$ (see Definition 
2.1) is only a subdomain of the whole domain of the uniform Lopatinski condition. Although, it should be noted that for nonlinear analysis (see section 4) a 1-symmetrizer has no advantages in comparison with a 2 -symmetrizer.

3. The constant coefficients linear analysis. In the rest of the paper we consider shock waves and only make certain remarks concerning the case of characteristic discontinuities.

Assumption 3.1. For the boundary conditions $(2.2 \mathrm{~b})$, rank $\widehat{B}=n$, i.e., the vectors $\left[\mathcal{P}^{0}(\widehat{\mathbf{U}})\right],\left[\mathcal{P}^{k}(\widehat{\mathbf{U}})\right], k=\overline{2, n}$, are linearly independent.

It follows from Assumption 3.1 that $n \leq N$ and there is a nonsingular matrix $\mathcal{M}=\mathcal{M}\left(\widehat{\mathbf{U}}^{+}, \widehat{\mathbf{U}}^{-}\right)$of order $N$ such that

$$
\mathcal{M B}=\left(\begin{array}{c}
I_{n} \\
0
\end{array}\right) .
$$

Let

$$
\mathcal{M}=\left(\begin{array}{c}
\mathcal{M}^{\mathrm{I}} \\
\mathcal{M}^{\mathrm{II}}
\end{array}\right)
$$

where $\mathcal{M}^{\mathrm{I}}$ and $\mathcal{M}^{\mathrm{II}}$ are matrices of order $n \times N$ and $(N-n) \times N$, respectively. Then the boundary conditions $(2.2 \mathrm{~b})$ can be divided into the two groups

$$
\begin{gathered}
\mathbf{F}=\mathcal{M}^{\mathrm{I}}\left[\widehat{S}^{-1} \widehat{A}_{\nu} \mathbf{U}\right]+\mathcal{M}^{\mathrm{I}} \mathbf{g}, \quad x_{1}=0, \\
-\mathcal{M}^{\mathrm{I}}\left[\widehat{S}^{-1} \widehat{A}_{\nu} \mathbf{U}\right]=\mathcal{M}^{\mathrm{II}} \mathbf{g}, \quad x_{1}=0 .
\end{gathered}
$$

Note that by cross differentiation one can, in principle, eliminate the front $f$ from relations (3.1). Such a procedure results in first order boundary conditions (see, e.g., (2.19)).

Assumption 3.1 is quite natural because, as was proved in [37], it is fulfilled for uniformly stable shock waves, i.e., when (2.2) satisfies the uniform Lopatinski condition. Moreover, as was shown in [17], Assumption 3.1 is also fulfilled for weakly stable shocks under some additional supposition. At the same time, for example, for gas dynamical and MHD shock waves Assumption 3.1 is always satisfied if only $R=\hat{\rho}^{+} / \hat{\rho}^{-} \neq 1$.

THEOREM 3.1. Suppose the Lax shock conditions (1.20) and all the assumptions above are fulfilled. Suppose also that (2.2) has a strictly dissipative p-symmetrizer. Then, the a priori estimate

$$
\begin{aligned}
\sum_{ \pm}\left\{\|\mathbf{U}(t)\| \|_{W_{2}^{p}\left(\mathbb{R}_{ \pm}^{n}\right)}\right. & \left.+\left\|\mathbf{U}^{ \pm}\right\|_{W_{2}^{p}\left([0, T] \times \mathbb{R}^{n-1}\right)}\right\}+\|f\|_{W_{2}^{p+1}\left([0, T] \times \mathbb{R}^{n-1}\right)} \\
\leq C\left\{\sum_{ \pm}\{\right. & \left.\left\|\mathbf{f}^{ \pm}\right\|_{W_{2}^{p}\left([0, T] \times \mathbb{R}_{ \pm}^{n}\right)}+\left\|\mathbf{U}_{0}\right\|_{W_{2}^{p}\left(\mathbb{R}_{ \pm}^{n}\right)}\right\} \\
& \left.+\|\mathbf{g}\|_{W_{2}^{p}\left([0, T] \times \mathbb{R}^{n-1}\right)}+\left\|f_{0}\right\|_{W_{2}^{p+1}\left(\mathbb{R}^{n-1}\right)}\right\}
\end{aligned}
$$

holds for any $t \in(0, T)$. Here $T$ is a positive constant, $C=C(T)$ is a positive constant independent of the initial data and the source terms,

$$
\|(\cdot)(t)\|_{W_{2}^{k}}^{2}:=\sum_{j=0}^{k}\left\|\partial_{t}^{j}(\cdot)(t)\right\|_{W_{2}^{k-j}}^{2} .
$$


If (2.2) has a dissipative (but not strictly dissipative) p-symmetrizer, the following weaker a priori estimate holds

$$
\begin{gathered}
\sum_{ \pm}\left\{\|\mathbf{U}(t)\|\left\|_{W_{2}^{r}\left(\mathbb{R}_{ \pm}^{n}\right)}+\right\| \mathbf{U}^{ \pm} \|_{W_{2}^{r-1}\left([0, T] \times \mathbb{R}^{n-1}\right)}\right\}+\|f\|_{W_{2}^{r}\left([0, T] \times \mathbb{R}^{n-1}\right)} \\
\leq C\left\{\sum_{ \pm}\left\{\left\|\mathbf{f}^{ \pm}\right\|_{W_{2}^{r}\left([0, T] \times \mathbb{R}_{ \pm}^{n}\right)}+\left\|\mathbf{U}_{0}\right\|_{W_{2}^{r}\left(\mathbb{R}_{ \pm}^{n}\right)}\right\}\right. \\
\left.+\|\mathbf{g}\|_{W_{2}^{r+1}\left([0, T] \times \mathbb{R}^{n-1}\right)}+\left\|f_{0}\right\|_{W_{2}^{r}\left(\mathbb{R}^{n-1}\right)}\right\}
\end{gathered}
$$

where $r=1$ for $p=0$ and $r=p$ for $p \geq 1$.

Proof. We will not prove estimate (3.3) in detail since arguments to do this are quite standard. By virtue of (2.6), (2.8), it follows from (2.5) that

$$
\begin{aligned}
& I_{1}(t)+\int_{0}^{t} \int_{\mathbb{R}^{n-1}}\left(\left|\mathbf{W}^{+}\right|^{2}+\left|\mathbf{W}^{-}\right|^{2}\right) d \mathbf{x}^{\prime} d t \\
& \quad \leq C_{1}\left\{I_{1}(0)+J(T)+\int_{0}^{t} I_{1}(s) d s\right\},
\end{aligned}
$$

where

$$
I_{1}(t)=\sum_{ \pm}\|\mathbf{W}(t)\|_{L_{2}\left(\mathbb{R}_{ \pm}^{n}\right)}^{2}, \quad J(T)=\|\mathbf{g}\|_{W_{2}^{p}\left([0, T] \times \mathbb{R}^{n-1}\right)}^{2}+\sum_{ \pm}\left\|\mathbf{f}^{ \pm}\right\|_{W_{2}^{p}\left([0, T] \times \mathbb{R}_{ \pm}^{n}\right)}^{2} .
$$

Here and below $C_{i}=C_{i}\left(\widehat{\mathbf{U}}^{+}, \widehat{\mathbf{U}}^{-}, \boldsymbol{\sigma}\right), i=1,2,3, \ldots$, appearing under the analysis of (2.2) are positive constants.

If $p \neq 0$, we use the elementary inequality

$$
I_{0}(t) \leq I_{0}(0)+\int_{0}^{t} I(s) d s
$$

coming from the trivial identity

$$
\frac{d}{d t} I_{0}(t)=2 \sum_{ \pm} \int_{\mathbb{R}_{ \pm}^{n}}\left(\mathbf{Y}, \mathbf{Y}_{t}\right) d t
$$

where $I(t)=I_{0}(t)+I_{1}(t), \mathbf{Y}=\left(\mathbf{W}_{0}, \ldots, \mathbf{W}_{p-1}\right), p \geq 1$,

$$
I_{0}(t)=\sum_{ \pm}\|\mid \mathbf{U}(t)\|_{W_{2}^{p-1}\left(\mathbb{R}_{ \pm}^{n}\right)}^{2} .
$$

Inequalities (3.5) and (3.6) yield

$$
I(t) \leq C_{1}\left\{I(0)+J(T)+\int_{0}^{t} I(s) d s\right\} .
$$

Applying Gronwall's lemma, one gets

$$
I(t) \leq C_{2}(I(0)+J(T)), \quad 0 \leq t \leq T .
$$

From trace's property one has

$$
\int_{0}^{t} \int_{\mathbb{R}^{n-1}}\left(\left|\mathbf{Y}^{+}\right|^{2}+\left|\mathbf{Y}^{-}\right|^{2}\right) d \mathbf{x}^{\prime} d t \leq \int_{0}^{t} I(s) d s .
$$


Adding up (3.5) and (3.8) and taking into account estimate (3.7), we obtain

$$
\sum_{ \pm}\left\|\mathbf{U}^{ \pm}\right\|_{W_{2}^{p}\left([0, T] \times \mathbb{R}^{n-1}\right)}^{2} \leq C_{3}(I(0)+J(T)) .
$$

Using (3.9), the boundary conditions (3.1), and an elementary inequality for $f$ like that in (3.6) for $\mathbf{U}$, we estimate the front perturbation $f$ :

$$
\|f\|_{W_{2}^{p+1}\left([0, T] \times \mathbb{R}^{n-1}\right)}^{2} \leq C_{4}\left\{I(0)+J(T)+\left\|f_{0}\right\|_{W_{2}^{p+1}\left(\mathbb{R}^{n-1}\right)}^{2}\right\} .
$$

Estimates (3.7), (3.9), and (3.10) imply the desired a priori estimate (3.3).

Let us now assume (2.2) has a dissipative (but not strictly dissipative) $p$-symmetrizer. If $p=0$ we differentiate system (2.5) with respect to $t$ and $\mathbf{x}^{\prime}$ and obtain a symmetric hyperbolic system for the vector $\left(\mathbf{U}_{t}, \mathbf{U}_{x_{2}}, \ldots, \mathbf{U}_{x_{n}}\right)$. Using this system for the case $p=0$ or system (2.5) for $p \geq 1$ and taking into account (2.6) and (2.7), we obtain the inequality

$$
I_{1}(t)+\int_{0}^{t} \int_{\mathbb{R}^{n-1}}(\widehat{B} \mathcal{G}, \widetilde{\mathbf{Z}}) d \mathbf{x}^{\prime} d t \leq C_{4}\left\{I_{1}(0)+J(T)+\int_{0}^{t} I_{1}(s) d s\right\},
$$

where $\widehat{B}$ is a constant matrix, $\widetilde{\mathbf{Z}}=\left(\mathbf{Z}^{+}, \mathbf{Z}^{-}\right), \mathbf{Z}=\left(\partial_{t} \mathbf{W}_{r-1}, \partial_{2} \mathbf{W}_{r-1}, \ldots, \partial_{n} \mathbf{W}_{r-1}\right)$ $(r=1$ for $p=0$ and $r=p$ for $p \geq 1)$. Other notations are the same as in (3.5), but for the case of 0 -symmetrizer $W:=W_{1}, p=1$ in $J$, and the vector $\mathcal{G}$ is formed by $\partial_{t, \mathrm{x}^{\prime}}^{\alpha} \mathbf{g}(|\alpha|=1)$ and $\left.\mathbf{f}^{ \pm}\right|_{x_{1}= \pm 0}$. While obtaining (3.11) we used the relations

$$
\mathbf{U}_{x_{1}}=-\left(\widehat{A}_{\nu}^{ \pm}\right)^{-1} \widehat{A}_{0}^{ \pm} \mathbf{U}_{t}-\sum_{k=2}^{n}\left(\widehat{A}_{\nu}^{ \pm}\right)^{-1} \widehat{A}_{k}^{ \pm} \mathbf{U}_{x_{k}}+\left(\widehat{A}_{\nu}^{ \pm}\right)^{-1} \mathbf{f}^{ \pm}, \quad \mathbf{x} \in \mathbb{R}_{ \pm}^{n}
$$

(recall that we consider shock waves and, therefore, $\operatorname{det} \widehat{A}_{\nu}^{ \pm} \neq 0$ ).

To estimate the boundary integral in the left-hand side of inequality (3.11) we carry out standard manipulations with derivatives. For example, with the terms like $u_{x_{2}}^{+} g$ and $u_{x_{2}}^{+} h^{+}$appearing in this integral we proceed as follows:

$$
\begin{gathered}
\int_{\mathbb{R}^{n-1}} u_{x_{2}}^{+} g d \mathbf{x}^{\prime}=-\int_{\mathbb{R}^{n-1}} u^{+} g_{x_{2}} d \mathbf{x}^{\prime}, \\
\int_{\mathbb{R}^{n-1}} u_{x_{2}}^{+} h^{+} d \mathbf{x}^{\prime}=-\int_{\mathbb{R}_{+}^{n}}\left(u_{x_{2}} h\right)_{x_{1}} d \mathbf{x}=\int_{\mathbb{R}_{+}^{n}}\left(u_{x_{1}} h_{x_{2}}-u_{x_{2}} h_{x_{1}}\right) d \mathbf{x},
\end{gathered}
$$

where $u=\partial^{\alpha} u_{j}, h=\partial^{\beta} f_{k}, g=\partial_{t, \mathrm{x}^{\prime}}^{\gamma} g_{k},|\alpha|=|\beta|=r-1,|\gamma|=r$, and $g_{k}$ and $f_{k}$ are, respectively, components of the vectors $\mathbf{g}$ and $\mathbf{f}^{+}$. Analogous standard arguments were also applied in [45] to treat lower order terms in the boundary integral for the variable coefficients linearized problem for current-vortex sheets. Observe that while estimating integrals like $\int_{0}^{t} \int_{\mathbb{R}^{n-1}} u_{t}^{+} h^{+} d \mathbf{x}^{\prime} d t$ we should be more careful because terms in the form $\partial_{t}\{\cdots\}$ do not disappear under the integration over the domain $[0, t] \times\left(\mathbb{R}_{+}^{n} \cup \mathbb{R}_{-}^{n}\right)$ (for corresponding simple arguments see [45]). As a result, omitting details, from (3.11) we deduce estimate (3.4).

Remark 3.1. Using

$$
\mathbf{U}_{t}=-\left(\widehat{A}_{0}^{ \pm}\right)^{-1} \widehat{A}_{\nu}^{ \pm} \mathbf{U}_{x_{1}}-\sum_{k=2}^{n}\left(\widehat{A}_{0}^{ \pm}\right)^{-1} \widehat{A}_{k}^{ \pm} \mathbf{U}_{x_{k}}+\left(\widehat{A}_{0}^{ \pm}\right)^{-1} \mathbf{f}^{ \pm}, \quad \mathbf{x} \in \mathbb{R}_{ \pm}^{n},
$$


one can reduce estimates (3.3) and (3.4) to those with the norms $\|(\cdot)(t)\|$ instead of the norms $\|(\cdot)(t)\|$.

COROLLARY 3.2. If the symmetric hyperbolic system (1.5) meets either the block structure condition [1,35] or the conditions of Métivier and Zumbrun [38] and problem (2.2) for the case of Lax shocks has a strictly dissipative p-symmetrizer, then in the parameter domain $D$ the boundary conditions (2.2b) satisfy the uniform Lopatinski condition.

Proof. First of all, following arguments like those used in [34] for strictly dissipative boundary value problems, one can easily obtain an analogue of estimate (3.3) when the exponentially weighted $W_{2, \eta}^{s}$-norms (with $s=p$ and $s=p+1$ ) are used instead of the usual Sobolev norms, where

$$
\|\cdot\|_{W_{2, \eta}^{s}}:=\sum_{|\alpha| \leq s} \eta^{s-|\alpha|}\left\|e^{-\eta t} \partial^{\alpha}(\cdot)\right\|_{L_{2}} .
$$

For the case when system (1.5) satisfies the block structure condition [1, 35, 32], it was proved in [32] (see also [37]) that such an estimate (with $s=0$ and $s=1$ ) holds for problem (2.2) if and only if this problem meets the uniform Lopatinski condition. This result was recently extended by Métivier and Zumbrun to the case of variable multiplicities provided that some additional conditions [38] hold. It is clear that the $L_{2, \eta}$-estimate (with the $W_{2, \eta}^{1}$-norm for $f$ ) implies $W_{2, \eta}^{s}$-estimates (see [37]). Hence, the boundary conditions $(2.2 \mathrm{~b})$ satisfy the uniform Lopatinski condition.

Remark 3.2. If the linear problem (2.2) meets the uniform Lopatinski condition and the symmetric hyperbolic system (1.5) satisfies either the block structure condition $[1,35]$ or the conditions of Métivier and Zumbrun [38], then the solution to (2.2) obeys an a priori $L_{2}$-estimate $[32,37]$. That is, if $p>0$, the result of Theorem 3.1 obtained for the linearized problem by the energy method is weaker than that in $[32,37]$ obtained by Kreiss' symmetrizer analysis in the sense that in estimate (3.3) we require more regularity for $\mathbf{U}$. However, if $p<[n / 2]+2$, for the original nonlinear problem the energy method gives the same result (see Theorem 4.1) as the technique used in $[32,37]$. Since $p<[n / 2]+2$ for all the known concrete examples of $p$-symmetrizers, we will suppose that this condition is satisfied.

Remark 3.3. In [45] the a priori estimates for the linearized problem for currentvortex sheets were written out for the case of the homogenous problem $\left(\mathbf{f}^{ \pm}=0\right.$ and $\mathrm{g}=0$ ). For the case of the nonhomogenous problem (2.2a), (2.16) (with the source term $\mathbf{g}$ in (2.16)), the a priori estimate

$$
\begin{gathered}
\sum_{ \pm}\left\{\left.\|\mathbf{U}(t)\|\right|_{\widetilde{W}_{2}^{1}\left(\mathbb{R}_{ \pm}^{3}\right)}+\left\|\mathbf{V}^{ \pm}\right\|_{L_{2}\left([0, T] \times \mathbb{R}^{2}\right)}\right\}+\|f\|_{W_{2}^{1}\left([0, T] \times \mathbb{R}^{2}\right)} \\
\leq C\left\{\sum_{ \pm}\left\{\left\|\mathbf{f}^{ \pm}\right\|_{W_{2}^{1}\left([0, T] \times \mathbb{R}_{ \pm}^{3}\right)}+\left\|\mathbf{U}_{0}\right\|_{\widetilde{W}_{2}^{1}\left(\mathbb{R}_{ \pm}^{3}\right)}\right\}\right. \\
\left.+\|\mathbf{g}\|_{W_{2}^{2}\left([0, T] \times \mathbb{R}^{2}\right)}+\left\|f_{0}\right\|_{W_{2}^{1}\left(\mathbb{R}^{n-1}\right)}\right\}
\end{gathered}
$$

can be deduced, provided that the sufficient neutral stability condition (2.18) holds. Here $\mathbf{V}\left(=\left(q, v_{1}, H_{1}\right)\right.$, see [45]) is the "noncharacteristic part" of $\mathbf{U}$,

$$
\|\mathbf{U}(t)\|_{\widetilde{W}_{2}^{s}\left(\mathbb{R}_{ \pm}^{n}\right)}^{2}=\|\mathbf{V}(t)\|_{W_{2}^{s}\left(\mathbb{R}_{ \pm}^{n}\right)}^{2}+\sum_{|\alpha| \leq s}\left\|\left(\partial_{t, \mathrm{x}^{\prime}}^{\alpha} \mathbf{U}\right)(t)\right\|_{L_{2}\left(\mathbb{R}_{ \pm}^{n}\right)}^{2} .
$$


For the general case of characteristic discontinuities, if we require that in (3.1)

$$
\mathcal{M}^{\mathrm{I}}\left[\widehat{S}^{-1} \widehat{A}_{\nu} \mathbf{U}\right]=\mathcal{M}_{1} \mathbf{V}^{+}+\mathcal{M}_{2} \mathbf{V}^{-}
$$

with corresponding matrices $\mathcal{M}_{i}$, i.e., the vector-function $\mathbf{F}$ can be expressed by the "noncharacteristic parts" $\mathbf{V}^{ \pm}$of the traces $\mathbf{U}^{ \pm}$, then in the counterparts of estimates (3.3) and (3.4) the $\widetilde{W}_{2}^{s}\left(\mathbb{R}_{ \pm}^{n}\right)$-norms are used instead of the $W_{2}^{s}\left(\mathbb{R}_{ \pm}^{n}\right)$-norms, and we can control only the "noncharacteristic" traces $\mathbf{V}^{ \pm}$. If assumption (3.12) does not hold, we have weaker a priori estimates. For example, this is so for current-vortex sheets if $\widehat{\mathbf{H}}^{+} \times \widehat{\mathbf{H}}^{-}=0$. For this case, the a priori estimate indicates already the loss of two derivatives from the front $f$ (see [45]).

4. Local existence of shock-front solutions. The local existence theorem for the nonlinear problem (1.17) has been first proved by Blokhin $[7,9]$ for uniformly stable gas dynamical shock waves by the direct energy method. Recall that the linearized constant coefficients problem for them has a strictly dissipative 2-symmetrizer (see section 2). The functional setting in the theorem from [7, 9] (see also [13]) is provided by the usual Sobolev spaces $W_{2}^{s}$, where $s \geq 3$. The analogous theorem, but in the exponentially weighted Sobolev spaces $W_{2, \eta}^{s}$, where $s$ is large enough, was proved by Majda [33] for Lax shocks by Kreiss' symmetrizer technique [27] and using pseudodifferential calculus, provided that the symmetric hyperbolic system satisfies the block structure condition $[1,35,32]$.

Recently, the theorem from [33] (see also [34]) was considerably improved by Métivier in [37], where the nonlinear local existence theorem was formulated in the form of Blokhin's theorem from [7, 9, 13] (see below). Actually, the theorem proved in [37] is valid for shock waves for which the linearized problem admits constructing Kreiss' symmetrizer. That is, the class of hyperbolic symmetrizable systems covered by this theorem is wider than that of systems satisfying the block structure condition. Moreover, taking into account the recent result in [38] mentioned above, the local existence theorem from [37] (see also [7, 9, 13]) takes place for the hyperbolic symmetrizable systems satisfying either the block structure condition or Métivier and Zumbrun's conditions [38]. That is, for Lax shock waves for which the assumption of Corollary 3.2 is fulfilled we have the following theorem (cf. $[7,9,13,37]$ ).

THEOREM 4.1. Let that the linearized constant coefficients problem (2.2) has a strictly dissipative p-symmetrizer. Suppose the initial data $(1.17 \mathrm{c})$ satisfy the hyperbolicity condition $A_{0}>0$ (for $\mathbf{x} \in \mathbb{R}_{ \pm}^{n}$ ), the Lax shock conditions (1.20), and the compatibility conditions (see [37]). Suppose also that $\left(\left.\mathbf{U}_{0}\right|_{x_{1}>0},\left.\mathbf{U}_{0}\right|_{x_{1}<0}, f_{0}\right) \in D$ for all $\mathbf{x} \in \mathbb{R}_{ \pm}^{n}$ (see Definition 2.1). Then, for all

$$
\left(\mathbf{U}_{0}, f_{0}\right) \in\left\{W_{2}^{s}\left(\mathbb{R}_{+}^{n}\right) \cap W_{2}^{s}\left(\mathbb{R}_{-}^{n}\right)\right\} \times W_{2}^{s+1}\left(\mathbb{R}^{n-1}\right),
$$

where $s \geq[n / 2]+2$, there is a sufficiently short time $T>0$ such that (1.17) has a unique solution

$$
(\mathbf{U}, f) \in Z_{T}^{s}=\left\{X_{s}\left([0, T], \mathbb{R}_{+}^{n}\right) \cap X_{s}\left([0, T], \mathbb{R}_{-}^{n}\right)\right\} \times W_{2}^{s+1}\left([0, T] \times \mathbb{R}^{n-1}\right),
$$

where

$$
X_{k}\left([0, T], \mathbb{R}_{ \pm}^{n}\right):=\bigcap_{j=0}^{k} C^{j}\left([0, T], W_{2}^{k-j}\left(\mathbb{R}_{ \pm}^{n}\right)\right)
$$

with the norm $\|\cdot\|_{X_{k}}=\max _{t \in[0, T]}|\|(\cdot)(t)\||_{W_{2}^{k}}$. 
It seems that for most physical examples of hyperbolic symmetrizable systems either the block structure condition or the "nonglancing" condition of Métivier and Zumbrun [38] is satisfied. Moreover, we still do not know any concrete example of a strictly dissipative $p$-symmetrizer for a hyperbolic system for which both of these conditions are violated. Therefore, in view of Corollary 3.2, construction of a strictly dissipative $p$-symmetrizer can be considered as an indirect test of the uniform Lopatinski condition. That is, as soon as such a symmetrizer is found, we have Theorem 4.1. In the light of this, there is now no practical sense for proving Theorem 4.1 directly by the energy method (as was earlier done in $[7,9,10]$ for gas dynamical shocks), i.e., without referring to $[37,38]$ and Corollary 3.2 .

We connect further perspectives of the method of $p$-symmetrizers with "nonstandard" problems, in particular, for characteristic discontinuities for which the structure of the Lopatinski determinant cannot be analyzed for technical reasons (see discussion in section 5). At the same time, to demonstrate how the energy method works for Lax shock waves for the case of variable coefficients and for the original nonlinear problem, we now outline the proof of Theorem 4.1. The main attention will be given to the deduction of an a priori estimate for the variable coefficients linearized problem $(1.24),(1.17 \mathrm{c})$. After that, to show the existence of solutions to $(1.24),(1.17 \mathrm{c})$ we comment how to go back from the system for $p$-derivatives with strictly dissipative boundary conditions to the original problem (1.24), (1.17c). At last, the proof of the existence of solutions to the nonlinear problem (1.17) follows from a fixed-point argument and we sketch it in the end of this section.

In the following we suppose that $p<[n / 2]+2$ (see Remark 3.2). We just observe that if we prove Theorem 4.1 by the energy method, then for the case $p \geq[n / 2]+2$ we have to assume that $s \geq \max \{[n / 2]+2, p+1\}$. We underline once more that we do not know concrete examples of $p$-symmetrizers with $p \geq[n / 2]+2$.

We first analyze the variable coefficients linear problem (1.24). We introduce the $\operatorname{norm}$ of $\left(\mathbf{u}(t, \mathbf{x}), \varphi\left(t, \mathbf{x}^{\prime}\right)\right) \in \mathbb{R}^{N} \times \mathbb{R}$ :

$$
\mathcal{N}_{T}^{k}(\mathbf{u}, \varphi):=\sum_{ \pm}\left\{\|\mathbf{u}\|_{X_{k}\left([0, T], \mathbb{R}_{ \pm}^{n}\right)}+\left\|\mathbf{u}^{ \pm}\right\|_{W_{2}^{k}\left([0, T] \times \mathbb{R}^{n-1}\right)}\right\}+\|\varphi\|_{W_{2}^{k+1}\left([0, T] \times \mathbb{R}^{n-1}\right)},
$$

where $k$ is a nonnegative integer number. Fix an integer $s \geq[n / 2]+2$ and consider $(\widehat{\mathbf{U}}, \hat{f}) \in Z_{T}^{s}$ with a time $T>0$. Assume that there is a constant $M>0$ such that

$$
\mathcal{N}_{T}^{s}(\widehat{\mathbf{U}}, \hat{f}) \leq M
$$

THEOREM 4.2. Given an integer $m \geq p$, suppose that problem (1.24) with "frozen" coefficients

$$
\left(\left.\widehat{\mathbf{U}}\right|_{x_{1}>0},\left.\widehat{\mathbf{U}}\right|_{x_{1}<0}, \widehat{\mathbf{F}}\right)=\left(\widehat{\mathbf{U}}^{+}, \widehat{\mathbf{U}}^{-}, \boldsymbol{\sigma}\right)
$$

has a strictly dissipative $p$-symmetrizer $(p<[n / 2]+2)$ and $(\widehat{\mathbf{U}}, \hat{f}) \in Z_{T}^{s}$, with $s=$ $\max \{m,[n / 2]+2\}$. Suppose also that the Lax shock conditions (1.20) and inequality (4.1) are fulfilled. Then, the following a priori estimate holds for the initial-boundaryvalue problem $(1.24),(1.17 \mathrm{c})$ :

$$
\begin{gathered}
\mathcal{N}_{T}^{m}(\mathbf{U}, f) \leq C(T, M)\left\{\sum_{ \pm}\left\{\left\|\mathbf{f}^{ \pm}\right\|_{W_{2}^{m}\left([0, T] \times \mathbb{R}_{ \pm}^{n}\right)}+\left\|\mathbf{U}_{0}\right\|_{W_{2}^{m}\left(\mathbb{R}_{ \pm}^{n}\right)}\right\}\right. \\
\left.+\|\mathbf{g}\|_{W_{2}^{m}\left([0, T] \times \mathbb{R}^{n-1}\right)}+\left\|f_{0}\right\|_{W_{2}^{m+1}\left(\mathbb{R}^{n-1}\right)}\right\} .
\end{gathered}
$$


Here and below $C=C(T, M), C_{i}=C_{i}(T, M), i=1,2,3, \ldots$, are positive constants independent of the data and depending on $T$ and $M$.

Proof. The methods for deducing the a priori estimate (4.2) are standard and based on the application of the Gagliardo-Nirenberg inequalities (see, e.g., [34])

$$
\begin{gathered}
\left\|\partial^{\alpha} u\right\|_{L_{2 p}(\Omega)} \leq c_{k}\|u\|_{L_{\infty}(\Omega)}^{1-1 / p}\|u\|_{W_{2}^{k}(\Omega)}^{1 / p}, \quad \frac{1}{p}=\frac{|\alpha|}{k} \\
\left\|\partial^{\alpha} u\right\|_{L_{p}(\Omega)} \leq c_{k}\|u\|_{L_{2}(\Omega)}^{1-r}\|u\|_{W_{2}^{k}(\Omega)}^{r}, \quad \frac{|\alpha|}{k}<r<1, \quad \frac{1}{p}=\frac{1}{2}+\frac{|\alpha|-r k}{\operatorname{dim} \Omega},
\end{gathered}
$$

where $c_{k}>0$ is a constant, $2<p<\infty$. The domain $\Omega$ can be, for example, $\mathbb{R}^{n}, \mathbb{R}_{ \pm}^{n}$, $[0, T] \times \mathbb{R}_{ \pm}^{n}$, or $[0, T] \times \mathbb{R}^{n-1}$ (in general, $\Omega$ is a Lipschitz domain).

Inequalities (4.3) and (4.4) imply a number of calculus inequalities (see, e.g., $[47,34])$. In particular, using (4.3) and (4.4), one can obtain the inequality

$$
\|u v\|_{W_{2}^{k}(\Omega)} \leq c_{k}\|u\|_{W_{2}^{q}(\Omega)}\|v\|_{W_{2}^{k}(\Omega)}, \quad q=\max \left\{\left[\frac{n}{2}\right]+1, k\right\}
$$

(here $\operatorname{dim} \Omega=n$ ). In Appendix $\mathrm{B}$ of [41] the following generalization of the last inequality was proved:

$$
\|(u v)(t)\|_{W_{2}^{k}(\Omega)} \leq c_{k}\|\| u(t)\|\|_{W_{2}^{q}(\Omega)}\|v(t)\|_{W_{2}^{k}(\Omega)},
$$

where $\Omega$ is a space domain (e.g., $\Omega=\mathbb{R}_{ \pm}^{n}$ ). It is clear that the analogous inequality holds when $x_{1}$ is fixed instead of $t$ :

$$
\left\langle\left\langle u v\left(x_{1}\right)\right\rangle\right\rangle_{k} \leq c_{k}\left\langle\langle \langle u ( x _ { 1 } ) \rangle \rangle _ { q } \left\langle\left\langle\left\langle v\left(x_{1}\right)\right\rangle\right\rangle_{k},\right.\right.
$$

where

$$
\left\langle\left\langle(\cdot)\left(x_{1}\right)\right\rangle\right\rangle_{k}:=\sum_{j=0}^{k}\left\|\partial_{1}^{j}(\cdot)\left(x_{1}\right)\right\|_{W_{2}^{k-j}\left([0, T] \times \mathbb{R}^{n-1}\right)}^{2} .
$$

One can also get the more special inequality

$$
\begin{aligned}
& \left\|\left(\partial^{\alpha_{1}} u_{1} \cdots \partial^{\alpha_{l}} u_{l}\right)\left(\partial_{t, \mathrm{x}^{\prime}}^{\beta} v\right)\left(x_{1}\right)\right\|_{L_{2}\left([0, T] \times \mathbb{R}^{n-1}\right)} \\
& \quad \leq c_{k}\left\|v\left(x_{1}\right)\right\|_{W_{2}^{k}\left([0, T] \times \mathbb{R}^{n-1}\right)} \prod_{i=1}^{l}\left\langle\left\langle u_{i}\left(x_{1}\right)\right\rangle\right\rangle_{q},
\end{aligned}
$$

where $\left|\alpha_{1}\right|+\cdots+\left|\alpha_{l}\right|+|\beta|=k$. To prove (4.8) we should follow arguments analogous to those from Appendix B of [41], and the proof is based mainly on the application of (4.4).

Let us obtain the system satisfied by the vector $\mathbf{W}_{\beta}=\partial_{t, \mathrm{x}^{\prime}}^{\beta} \mathbf{W}$, with $|\beta| \leq m-p$. It follows from (1.24a) that

$$
L(\widehat{\mathbf{U}}, \widehat{\mathbf{F}})\left(\partial^{\alpha^{i}} \partial_{t, \mathrm{x}^{\mathbf{\prime}}}^{\beta} \mathbf{U}\right)=\mathbf{f}_{i \beta}^{ \pm} \quad \text { if } \quad \mathbf{x} \in \mathbb{R}_{ \pm}^{n},
$$

where

$$
\mathbf{f}_{i \beta}^{ \pm}=\partial^{\alpha^{i}} \partial_{t, \mathbf{x}^{\prime}}^{\beta} \mathbf{f}^{ \pm}-\left[\partial^{\alpha^{i}} \partial_{t, \mathrm{x}^{\prime}}^{\beta}, L(\widehat{\mathbf{U}}, \widehat{\mathbf{F}})\right] \mathbf{U}
$$


Here and below we use the notation of commutator: $[a, b] c:=a(b c)-b(a c)$. From systems (4.9) with $i=\overline{1, d}$ we construct the system for $\mathbf{W}_{\beta}$ (cf. (2.4), (2.5)):

$$
\mathcal{L}(\widehat{\mathbf{U}}, \widehat{\mathbf{F}}) \mathbf{W}_{\beta}=\mathcal{F}_{\beta}^{ \pm}, \quad \mathbf{x} \in \mathbb{R}_{ \pm}^{n},
$$

where

$$
\begin{gathered}
\mathcal{L}=\mathcal{L}(\widehat{\mathbf{U}}, \widehat{\mathbf{F}})=\mathcal{A}_{0}(\widehat{\mathbf{U}}, \widehat{\mathbf{F}}) \partial_{t}+\sum_{j=1}^{n} \mathcal{A}_{j}(\widehat{\mathbf{U}}, \widehat{\mathbf{F}}) \partial_{j}, \quad \widetilde{\mathbf{f}}_{\beta}^{ \pm}=\left(\mathbf{f}_{1 \beta}^{ \pm}, \ldots, \mathbf{f}_{d \beta}^{ \pm}\right), \\
\mathcal{F}_{\beta}^{ \pm}=P(\widehat{\mathbf{U}}, \widehat{\mathbf{F}}) \widetilde{\mathbf{f}}_{\beta}^{ \pm}-\sum_{j=1}^{K} \sum_{|\alpha|=p} \mathbf{R}_{j, \alpha}(\widehat{\mathbf{U}}, \widehat{\mathbf{F}})\left[\partial^{\alpha} \partial_{t, \mathrm{x}^{\prime}}^{\beta}, \widehat{\mathbf{N}}\right] \partial_{1} \mathbf{\Psi}_{j}(\mathbf{U})
\end{gathered}
$$

the matrices $P(\widehat{\mathbf{U}}, \widehat{\mathbf{F}})$ and the vectors $\mathbf{R}_{j, \alpha}(\widehat{\mathbf{U}}, \widehat{\mathbf{F}})$ form the strictly dissipative $p$ symmetrizer $\mathbb{S}$ if we "freeze" their coefficients, and the matrices $\mathcal{A}_{i}(i=\overline{0, n})$ with "frozen" coefficients are the same as in (2.5).

For system (4.10) with variable coefficients, the counterpart of condition (2.8) is

$$
-\left.\left[\left(\mathcal{A}_{1}(\widehat{\mathbf{U}}, \widehat{\mathbf{F}}) \mathbf{W}_{\beta}, \mathbf{W}_{\beta}\right)\right]\right|_{x_{1}=0} \geq \delta\left(\left|\mathbf{W}_{\beta}^{+}\right|^{2}+\left|\mathbf{W}_{\beta}^{-}\right|^{2}\right)-\delta^{-1} g^{2},
$$

where $g^{2}$ is a sum of terms in the form

$$
\begin{aligned}
\left|G_{k}\left(\widehat{\mathbf{U}}^{+}, \widehat{\mathbf{U}}^{-}, \widehat{\mathbf{F}}\right) \partial_{t, \mathrm{x}^{\prime}}^{\alpha} \mathbf{g}\right|^{2}, & \left.\left|G_{l}\left(\widehat{\mathbf{U}}^{+}, \widehat{\mathbf{U}}^{-}, \widehat{\mathbf{F}}\right) \partial^{\gamma} \mathbf{f}^{ \pm}\right|_{x_{1}= \pm 0}\right|^{2}, \\
\left|G_{l_{0}}\left(\widehat{\mathbf{U}}^{+}, \widehat{\mathbf{U}}^{-}, \widehat{\mathbf{F}}\right) \partial^{\gamma} \mathbf{U}^{ \pm}\right|^{2}, & \text { and } \quad\left|G_{l_{0}}\left(\widehat{\mathbf{U}}^{+}, \widehat{\mathbf{U}}^{-}, \widehat{\mathbf{F}}\right) \partial_{t, \mathbf{x}^{\prime}}^{\gamma} \mathbf{F}\right|^{2},
\end{aligned}
$$

with

$$
\begin{gathered}
G_{i}\left(\widehat{\mathbf{U}}^{+}, \widehat{\mathbf{U}}^{-}, \widehat{\mathbf{F}}\right)=\partial^{\alpha_{1}} \hat{u}_{i_{1}}^{+} \cdots \partial^{\alpha_{j}} \hat{u}_{i_{j}}^{+} \partial^{\alpha_{j+1}} \hat{u}_{i_{j+1}}^{-} \cdots \partial^{\alpha_{r}} \hat{u}_{i_{r}}^{-} \\
\quad \times \partial_{t, \mathrm{x}^{\prime}}^{\alpha_{r+1}} \widehat{F}_{i_{r+1}} \cdots \partial_{t, \mathrm{x}^{\prime}}^{\alpha_{q}} \widehat{F}_{i_{q}} H\left(\widehat{\mathbf{U}}^{+}, \widehat{\mathbf{U}}^{-}, \widehat{\mathbf{F}}\right), \\
\left|\alpha_{1}\right|+\ldots+\left|\alpha_{q}\right|=i, \quad 0 \leq q \leq 2 N+n, \\
k+|\alpha| \leq m, \quad l+|\gamma| \leq m-1, \quad l_{0}+|\gamma| \leq m, \quad|\gamma| \leq m-1
\end{gathered}
$$

(for constant coefficients, cf. (2.8), $k=l=0$ and there are no lower order terms, i.e., $\left.G_{l_{0}} \equiv 0\right)$. Here $\widehat{F}_{i_{j}}$ is a component of the vector $\widehat{\mathbf{F}}\left(\widehat{F}_{i_{j}}=\hat{f}_{t}\right.$ or $\left.\widehat{F}_{i_{j}}=\hat{f}_{x_{k}}\right)$, $H\left(\widehat{\mathbf{U}}^{+}, \widehat{\mathbf{U}}^{-}, \widehat{\mathbf{F}}\right)$ is a matrix which elements are determined by the elements of the matrices $A_{0}, A_{\nu}$, and $A_{k}(k=\overline{2, n})$ and their derivatives up to order $m$ with respect to $\mathbf{U}$ and $\mathbf{F}$.

Since arguments below are standard we are quite brief in the rest of the proof. In view of (4.11), using arguments as in (3.6), (3.8) and applying energy methods to (4.10), we deduce the inequality

$$
I_{\tan }(t)+\int_{0}^{t} I_{\tan }^{\operatorname{tr}}(s) d s \leq C_{1}(T, M)\left\{I(0)+J(t)+\int_{0}^{t} I(s) d s\right\}
$$


where

$$
\begin{gathered}
I_{\tan }(t)=\sum_{ \pm}\left\{\|\mathbf{U}(t)\|_{W_{2}^{p}\left(\mathbb{R}_{ \pm}^{n}\right)}^{2}+\sum_{|\beta| \leq m-p}\left\|\mathbf{W}_{\beta}\right\|_{L_{2}\left(\mathbb{R}_{ \pm}^{n}\right)}^{2}\right\}, \\
I_{\tan }^{\operatorname{tr}}(t)=\sum_{ \pm}\left\{\sum_{j=0}^{p}\left\|\partial_{1}^{j} \mathbf{U}^{ \pm}(t)\right\|_{W_{2}^{p-j}\left(\mathbb{R}^{n-1}\right)}^{2}+\sum_{|\beta| \leq m-p}\left\|\mathbf{W}_{\beta}^{ \pm}\right\|_{L_{2}\left(\mathbb{R}^{n-1}\right)}^{2}\right\} \\
\partial_{1}^{j} \mathbf{U}^{ \pm}:=\left.\partial_{1}^{j} \mathbf{U}\right|_{x_{1}= \pm 0}, \quad I(t)=\sum_{ \pm} \mid\|\mathbf{U}(t)\|_{W_{2}^{m}\left(\mathbb{R}_{ \pm}^{n}\right)}^{2}, \\
J(t)=\|g\|_{L_{2}\left([0, t] \times \mathbb{R}^{n-1}\right)}^{2}+\sum_{ \pm} \sum_{|\beta| \leq m-p}\left\|\mathcal{F}_{\beta}^{ \pm}\right\|_{L_{2}\left([0, t] \times \mathbb{R}^{n}\right)}^{2} .
\end{gathered}
$$

The commutator $\left[\partial^{\alpha^{i}} \partial_{t, \mathrm{x}^{\prime}}^{\beta}, L(\widehat{\mathbf{U}}, \widehat{\mathbf{F}})\right] \mathbf{U}$ is a sum of terms $G_{k}(\widehat{\mathbf{U}}, \widehat{\mathbf{F}}) \partial^{\alpha} \mathbf{U}$, where $G_{k}$ are determined as $G_{i}$ above (but not on the boundary), $k+|\alpha| \leq m+1, k \geq 1$, and $|\alpha| \geq 1$. Since $k \geq 1$ and $|\alpha| \geq 1$, applying (4.6) with $k=m-1$ and using elementary inequalities like

$$
\sum_{\left|\alpha_{1}\right|+\ldots+\left|\alpha_{l}\right| \leq k}\left\|\partial^{\alpha_{1}} v_{1} \cdots \partial^{\alpha_{l}} v_{l}\right\|_{L_{2}} \leq \mathrm{const}\left\|v_{1} \cdots v_{l}\right\|_{W_{2}^{k}}
$$

one estimates the commutator

$$
\sum_{ \pm}\left\|\left[\partial^{\alpha^{i}} \partial_{t, \mathbf{x}^{\prime}}^{\beta}, L(\widehat{\mathbf{U}}, \widehat{\mathbf{F}})\right] \mathbf{U}\right\|_{L_{2}\left([0, t] \times \mathbb{R}^{n-1}\right)}^{2} \leq C_{2}(T, M) \int_{0}^{t} I(s) d s
$$

Then, estimating analogously other terms in $\mathcal{F}_{\beta}^{ \pm}$, one gets

$$
\sum_{ \pm} \sum_{|\beta| \leq m-p}\left\|\mathcal{F}_{\beta}^{ \pm}\right\|_{L_{2}\left([0, t] \times \mathbb{R}^{n-1}\right)}^{2} \leq C_{3}(T, M)\left\{\sum_{ \pm}\left\|\mathbf{f}^{ \pm}\right\|_{W_{2}^{m}\left([0, T] \times \mathbb{R}_{ \pm}^{n}\right)}^{2}+\int_{0}^{t} I(s) d s\right\} .
$$

To estimate the $L_{2}$-norm of $g$ we use inequality (4.7) at $x_{1}= \pm 0$ and trace's property. As a result, one has

$$
\begin{aligned}
\|g\|_{L_{2}\left([0, t] \times \mathbb{R}^{n-1}\right)}^{2} \leq C_{4}(T, M)\left\{\|\mathbf{g}\|_{W_{2}^{m}\left([0, T] \times \mathbb{R}^{n-1}\right)}^{2}\right. & \\
& \left.+\sum_{ \pm}\left\|\mathbf{f}^{ \pm}\right\|_{W_{2}^{m}\left([0, T] \times \mathbb{R}_{ \pm}^{n}\right)}^{2}+\int_{0}^{t}\left(I(s)+\|\mathbf{F}(s)\|_{W_{2}^{m-1}\left(\mathbb{R}^{n-1}\right)}^{2}\right) d s\right\} .
\end{aligned}
$$

Expressing $\mathbf{F}$ by $\mathbf{U}^{ \pm}$and $\mathbf{g}$ ((3.1) for variable coefficients is applied) and using trace's property, from (4.12) one obtains

$$
I_{\mathrm{tan}}(t)+\int_{0}^{t} I_{\tan }^{\mathrm{tr}}(s) d s \leq C_{5}(T, M)\left\{I(0)+J_{1}(T)+\int_{0}^{t} I(s) d s\right\}
$$

where

$$
J_{1}(T)=\|\mathbf{g}\|_{W_{2}^{m}\left([0, T] \times \mathbb{R}^{n-1}\right)}^{2}+\sum_{ \pm}\left\|\mathbf{f}^{ \pm}\right\|_{W_{2}^{m}\left([0, T] \times \mathbb{R}_{ \pm}^{n}\right)}^{2}
$$


Applying energy methods to systems for the vectors $\partial^{\alpha} \mathbf{U}$ with $|\alpha| \leq m$ and reasoning as above one can easily obtain the inequality

$$
I(t)-\int_{0}^{t} I^{\operatorname{tr}}(s) d s \leq C_{6}(T, M)\left\{I(0)+J_{1}(T)+\int_{0}^{t} I(s) d s\right\},
$$

where

$$
I^{\operatorname{tr}}(t)=\sum_{ \pm} \sum_{j=0}^{m}\left\|\partial_{1}^{j} \mathbf{U}^{ \pm}(t)\right\|_{W_{2}^{m-j}\left(\mathbb{R}^{n-1}\right)}^{2} .
$$

To get an inequality for $I(t)$ when "+" stands in (4.14) instead of "-", we use the great advantage that the boundary conditions are strictly dissipative and, therefore, one has the positive integral in the left-hand side of inequality (4.13). We should now estimate the "full trace" $\int_{0}^{t} I^{\operatorname{tr}}(s) d s$ by $\int_{0}^{t} I_{\tan }^{\operatorname{tr}}(s) d s$.

Using the equations

$$
\left.\mathbf{U}_{x_{1}}\right|_{x_{1}= \pm 0}=\left.A_{\nu}^{-1}(\widehat{\mathbf{U}}, \widehat{\mathbf{F}})\left\{\mathbf{f}^{ \pm}-A_{0}(\widehat{\mathbf{U}}) \mathbf{U}_{t}-\sum_{k=2}^{n} A_{k}(\widehat{\mathbf{U}}) \mathbf{U}_{x_{k}}\right\}\right|_{x_{1}= \pm 0}
$$

one has that $\partial^{\alpha} \mathbf{U}^{ \pm}$with $|\alpha|=m$ is a sum of terms $G_{k}\left(\widehat{\mathbf{U}}^{ \pm}, \widehat{\mathbf{F}}\right) \partial_{t, \mathrm{x}^{\prime}}^{\beta} \mathbf{U}^{ \pm}$and $G_{l}\left(\widehat{\mathbf{U}}^{ \pm}, \widehat{\mathbf{F}}\right)$ $\times\left.\partial^{\gamma} \mathbf{f}^{ \pm}\right|_{x_{1}= \pm 0}$, where $k+|\beta| \leq m$ and $l+|\gamma| \leq m-1$. Applying to these terms inequalities (4.8) and (4.7) (at $x_{1}= \pm 0$ ), respectively, one gets the desired estimate

$$
\int_{0}^{t} I^{\operatorname{tr}}(s) d s \leq C_{7}(T, M) \int_{0}^{t} I_{\tan }^{\operatorname{tr}}(s) d s .
$$

Summing up (4.13) multiplied by $2 C_{7}$ with (4.14) and using (4.15), we obtain

$$
I(t)+\int_{0}^{t} I^{\operatorname{tr}}(s) d s \leq C_{8}(T, M)\left\{I(0)+J_{1}(T)+\int_{0}^{t} I(s) d s\right\},
$$

where $C_{8}=C_{6}+2 C_{5} C_{7}$.

Throwing away the positive integral in the left-hand side of (4.16) and applying Gronwall's lemma yield

$$
I(t) \leq C_{9}(T, M)\left(I(0)+J_{1}(T)\right), \quad 0 \leq t \leq T .
$$

It follows from (4.16) and (4.17) that

$$
\sum_{ \pm}\left\|\mathbf{U}^{ \pm}\right\|_{W_{2}^{m}}^{2}\left([0, T] \times \mathbb{R}^{n-1}\right) \leq C_{10}(T, M)\left(I(0)+J_{1}(T)\right) .
$$

At last, using the boundary conditions (3.1) (for variable coefficients) and applying (4.5) with $\Omega=[0, T] \times \mathbb{R}^{n-1}$ and $k=m$, we get from (4.18) that

$$
\|f\|_{W_{2}^{m+1}\left([0, T] \times \mathbb{R}^{n-1}\right)}^{2} \leq C_{11}(T, M)\left\{I(0)+J_{1}(T)+\left\|f_{0}\right\|_{W_{2}^{m+1}\left(\mathbb{R}^{n-1}\right)}^{2}\right\} .
$$

Estimates (4.17)-(4.19) imply (4.2).

Remark 4.1. In [7, 9, 10], the estimate in the form of (4.2) was obtained for gas dynamical shock waves by an accurate use of various Sobolev's imbedding theorems. 
Unlike $[7,9,10]$, the proof of Theorem 4.2 above relies on the Gagliardo-Nirenberg inequalities and is, therefore, closer to arguments of Metivier [37]. But, if $\Omega=[0, T] \times \mathbb{R}_{ \pm}^{n}$ or $\Omega=[0, T] \times \mathbb{R}^{n-1}$ the constants in (4.3), (4.4) blow up as $T \rightarrow 0$. This unpleasant fact can prevent the proof of existence for the nonlinear problem. To overcome this difficulty it was suggested in [37] to use some substitutes of the Gagliardo-Nirenberg inequalities for which the constants are uniform with respect to $T$ as $T \rightarrow 0$ (we do not want to go into details and just refer to [37]). Using such substitutes allows one to prove some modifications of inequalities (4.5)-(4.8) which now include norms of $u(0)$ and $v(0)$ (see [37]). Further arguments in the proof of Theorem 4.2 remain valid (with little modification), and the constant $C(T, M)$ in (4.2) is uniform with respect to $T$ as $T \rightarrow 0$.

Consider the system

$$
\begin{gathered}
\widetilde{L}(\widehat{\mathbf{U}}, \widehat{\mathbf{F}}) \mathbf{Y}+C(\widehat{\mathbf{U}}, \widehat{\mathbf{F}}) \mathbf{Y}=\widetilde{\mathbf{f}}_{p-1}^{ \pm}, \\
\mathcal{L}(\widehat{\mathbf{U}}, \widehat{\mathbf{F}}) \mathbf{W}=\mathcal{F}^{ \pm}, \quad \mathbf{x} \in \mathbb{R}_{ \pm}^{n},
\end{gathered}
$$

where $\mathbf{Y}=\left(\mathbf{U}, \mathbf{W}_{1}, \ldots, \mathbf{W}_{p-1}\right)$, system (4.20a) is formed by (1.24a) and systems obtained by the differentiation of (1.24a) with respect to $t$ and $\mathbf{x}$; the matrix $C$ can be explicitly written out, $\widetilde{\mathbf{f}}_{p-1}^{ \pm}=\left(\partial^{\alpha^{1}} \mathbf{f}^{ \pm}, \ldots, \partial^{\alpha^{d_{0}}} \mathbf{f}^{ \pm}\right), d_{0}=C_{n+p-1}^{p-1}$, etc. (see section 2); system (4.20b) coincides with (4.10) for $|\beta|=0$. We supplement system (4.20) with the boundary conditions $(1.24 \mathrm{~b})$. All other boundary conditions follow from (1.24b) and system (4.20) itself at $x_{1}=0$.

System (4.20a) is equivalently rewritten as

$$
A_{0}(\widehat{\mathbf{U}}) \mathbf{Y}_{t} \mp \mathbf{Y}_{x_{1}}+\sum_{k=2}^{n} A_{k}(\widehat{\mathbf{U}}) \mathbf{Y}_{x_{k}}+C^{ \pm}(\widehat{\mathbf{U}}, \widehat{\mathbf{F}}) \mathbf{W}=\widetilde{\mathbf{f}}_{p-1}^{ \pm}, \quad \mathbf{x} \in \mathbb{R}_{ \pm}^{n}
$$

where $C^{ \pm} \mathbf{W}=C \mathbf{Y}+\left(A_{\nu} \pm I\right) \mathbf{Y}_{x_{1}}$. The boundary matrix for system (4.21), (4.20b) is $\operatorname{diag}\left(-I, A_{\nu}\right)$ for $x_{1}>0$ and $\operatorname{diag}\left(I, A_{\nu}\right)$ for $x_{1}<0$. Clearly, the boundary conditions for system (4.21), (4.20b) are strictly dissipative (see (4.11) for $|\beta|=0$ ).

Thus, system (4.21), (4.20b), that is equivalent to (4.20), has strictly dissipative boundary conditions. The initial-boundary-value problem for system (4.21), (4.20b) differs from one studied in Appendix A of [43] only by the presence of the unknown function $f$ in the boundary conditions. The compatibility conditions for (1.24) can be written by analogy with those for standard boundary conditions in [40, 43]. The existence of a smooth solution $\left(\mathbf{U}, \mathbf{W}_{1}, \ldots, \mathbf{W}_{p-1}, \mathbf{W}\right) \in W_{2}^{m-p}$ to the problem for system (4.21), (4.20b) is proved exactly in the same manner as in Appendix A of [43] for linear hyperbolic problems with strictly dissipative boundary conditions. Moreover, the component $\mathbf{U}$ of this solution satisfies the original problem (1.24). Note also that in [10] the existence of smooth solutions to the linearized problem for gas dynamical shock waves was proved by approximation by grid functions. Such an approach suggested by Godunov [25] for linear hyperbolic problems with strictly dissipative boundary conditions and applied by Blokhin [10] to gas dynamical shock waves can also be used for general Lax shocks under consideration. So, we have the following existence theorem. 
THEOREM 4.3. Let that all the assumptions of Theorem 4.2 are satisfied. Then, for all the data

$$
\begin{aligned}
& \mathbf{U}_{0} \in W_{2}^{m}\left(\mathbb{R}_{+}^{n}\right) \cap W_{2}^{m}\left(\mathbb{R}_{-}^{n}\right), \quad f_{0} \in W_{2}^{m+1}\left(\mathbb{R}^{n-1}\right), \\
& \mathbf{f}^{ \pm} \in W_{2}^{m}\left([0, T] \times \mathbb{R}_{ \pm}^{n}\right), \quad \mathbf{g} \in W_{2}^{m}\left([0, T] \times \mathbb{R}^{n-1}\right)
\end{aligned}
$$

satisfying the compatibility conditions up to order $m-1$, the initial-boundary-value problem $(1.24),(1.17 \mathrm{c})$ has a unique solution $(\mathbf{U}, f) \in Z_{T}^{m}$ that obeys the a priori estimate (4.2).

Sketch of the proof of Theorem 4.1. The proof follows from a fixed-point argument and we are quite brief here. For a time $T>0$, a constant $M>0$, and an integer $s \geq[n / 2]+2$, we define

$$
\begin{aligned}
\mathcal{K}=\left\{(\widehat{\mathbf{U}}, \hat{f}) \in Z_{T}^{s} \mid \mathcal{N}_{T}^{s}(\widehat{\mathbf{U}}, \hat{f}) \leq M, \widehat{\mathbf{U}}(0, \mathbf{x})=\mathbf{U}_{0}(\mathbf{x}), \hat{f}\left(0, \mathbf{x}^{\prime}\right)=f_{0}\left(\mathbf{x}^{\prime}\right)\right. & \\
& \left.\left(\mathbf{U}_{0}, f_{0}\right) \in\left\{\bigcap_{ \pm} W_{2}^{s}\left(\mathbb{R}_{ \pm}^{n}\right)\right\} \times W_{2}^{s+1}\left(\mathbb{R}^{n-1}\right) \text { is compatible to order } s-1\right\}
\end{aligned}
$$

We do not specify here the compatibility conditions and just refer to [37].

Consider now the mapping $\Lambda:(\widehat{\mathbf{U}}, \hat{f}) \rightarrow(\mathbf{U}, f)$, where $(\mathbf{U}, f)$ satisfies the initialboundary-value problem (1.24), (1.17c) with $\mathbf{f}^{ \pm} \equiv 0$ and

$$
\mathbf{g}=\left[\mathcal{P}^{1}(\widehat{\mathbf{U}})\right]-\left[S^{-1}(\widehat{\mathbf{U}}) A_{\nu}(\widehat{\mathbf{U}}, \widehat{\mathbf{F}}) \widehat{\mathbf{U}}\right] .
$$

Actually, with such a choice of $\mathbf{g}$ the linear conditions in (1.24b) are Newton's approximation of the nonlinear boundary conditions (1.17b) (see discussion in [34]). Theorem 4.3 guarantees the existence of $(\mathbf{U}, f) \in Z_{T}^{s}$. Moreover, it follows from estimate (4.2) that $\Lambda(\mathcal{K}) \subset \mathcal{K}$ for appropriate choices of $T$ and $M$ (see Remark 4.1).

Consider $\left(\widehat{\mathbf{U}}^{i}, \hat{f}^{i}\right) \in \mathcal{K}$ and let $(\mathbf{U}, f)=\Lambda\left(\widehat{\mathbf{U}}^{i}, \hat{f}^{i}\right), i=1,2$. For the differences $\mathbf{U}^{1}-\mathbf{U}^{2}$ and $f^{1}-f^{2}$ we obtain problem (1.24) with the trivial initial data, the coefficients $(\widehat{\mathbf{U}}, \hat{f})=\left(\widehat{\mathbf{U}}^{1}, \hat{f}^{1}\right)$,

$$
\mathbf{f}^{ \pm}=\left(L\left(\widehat{\mathbf{U}}^{2}, \widehat{\mathbf{F}}^{2}\right)-L\left(\widehat{\mathbf{U}}^{1}, \widehat{\mathbf{F}}^{1}\right)\right) \mathbf{U}^{2}, \quad \mathbf{x} \in \mathbb{R}_{ \pm}^{n},
$$

and a corresponding $\mathbf{g}$ (it can be easily written down as well). Applying estimate (4.2) with $m=s-1$ to this problem and using the mean-value theorem for $\mathbf{f}^{ \pm}$and $\mathrm{g}$, one gets

$$
\mathcal{N}_{T}^{s-1}\left(\mathbf{U}^{1}-\mathbf{U}^{2}, f^{1}-f^{2}\right) \leq \delta \mathcal{N}_{T}^{s-1}\left(\widehat{\mathbf{U}}^{1}-\widehat{\mathbf{U}}^{2}, \hat{f}^{1}-\hat{f}^{2}\right),
$$

where the positive constant $\delta=\delta(T, M)<1$ for $T$ sufficiently small (we do not describe in detail the choice of $T$ and $M$ and just refer to standard arguments, for example, in [34] for the Cauchy problem or in [41, 42] for initial-boundary-value problems).

That is, the mapping $\Lambda$ is a contraction in the low norm $\mathcal{N}_{T}^{s-1}$. Hence, there exists a unique fixed point $(\mathbf{U}, f)=(\widehat{\mathbf{U}}, \hat{f}) \in \mathcal{K}$ which solves $(1.17)$.

5. Concluding remarks. By introducing the notations of dissipative and strictly dissipative $p$-symmetrizers we have formalized the energy method applied earlier to strong discontinuities for concrete hyperbolic systems of conservation laws. We have proved that if the constant coefficients linearized problem for Lax shocks has 
a strictly dissipative $p$-symmetrizer, then under natural assumptions this implies the local in time existence of shock-front solutions of the original nonlinear system. This result recovers Blokhin's local existence theorem for gas dynamical shock waves [7, 9] and enables one to conclude the local existence of shock-front solutions for various concrete models (MHD [12], radiation hydrodynamics [3, 15], Landau's equations of superfluid [11], etc.) for which a priori estimates with no loss of derivatives for constant coefficients linearized problems were earlier deduced by the energy method.

It seems that the result of Theorem 4.1 could be extended, under appropriate assumptions, to the case of characteristic discontinuities. Note, however, that we do not know any concrete example of a characteristic discontinuity for which one can construct a strictly dissipative $p$-symmetrizer. Evidently, this is because all the known characteristic discontinuities (vortex sheets, current-vortex sheets, Alfvén discontinuities, etc.) can be only neutrally stable, i.e., the uniform Lopatinski condition is never satisfied for them.

With regard to the case where the loss of derivatives phenomenon takes place, i.e., when we are able to construct only a dissipative (but not strictly dissipative) $p$ symmetrizer, a theorem for the variable coefficients linearized problem like Theorem 4.3 could be proved both for Lax shocks and characteristic discontinuities. In the generic case, for characteristic discontinuities the functional setting is provided by the anisotropic weighted Sobolev spaces $H_{*}^{s}$ (see [42] and references therein). Concerning the proof of a local existence theorem, it seems that the only way to overcome difficulties connected with the loss of derivatives phenomenon is the use of the Nash-Moser method (see discussion in Remark 1.2). Note that the existence of a dissipative (but not strictly dissipative) $p$-symmetrizer implies the fulfillment of the (weak) Lopatinski condition, i.e., the weak stability of a corresponding strong discontinuity. In particular, the existence of a dissipative $p$-symmetrizer for a planar Lax shock implies the weak stability of this shock wave and, in view of the recent result of Coulombel and Secchi [20] (see Remark 1.2), the nonlinear existence of nonplanar shock waves that are close to the planar shock under consideration.

Unfortunately, there is not a general procedure to construct a $p$-symmetrizer. At the same time, if it was somehow constructed, we do not need to examine the Lopatinski condition, which is often untestable analytically (numerical testing is usually not so simple either). The requirements for a set $\mathbb{S}$ to be a (strictly) dissipative $p$-symmetrizer suggest sufficient or, sometimes, necessary and sufficient conditions for the fulfillment of the (uniform) Lopatinski condition. In this connection, the best example for illustration is the construction of the dissipative 0 -symmetrizer for current-vortex sheets [45] that first enabled the finding of wide sufficient conditions for their neutral stability (i.e., sufficient conditions of the macroscopic stability of the heliopause [4]).

For Lax shock waves, the construction of a strictly dissipative $p$-symmetrizer can be interpreted as an indirect test of the uniform Lopatinski condition and, referring then to $[37,38]$, we have at once the local existence theorem for the nonlinear problem. However, to construct Kreiss' symmetrizer for the case of characteristic discontinuities it is necessary to know not only that the Lopatinski condition is satisfied but also how it is satisfied, i.e., to know a detailed structure of the Lopatinski determinant (see [19]). For example, for current-vortex sheets [45] we know sufficient conditions for the fulfillment of the Lopatinski condition, but the structure of the Lopatinski determinant cannot be analyzed because of insuperable technical difficulties. That is, the only way to achieve a nonlinear result is to follow the energy method in the variable coefficients and nonlinear analysis as well. In the light of this, we think that future perspectives of 
the method of $p$-symmetrizers are connected with "nonstandard" problems for which either Kreiss' symmetrizer technique does not work for technical reasons or the general theory is still not developed (as, for example, for nonhyperbolic problems appearing for incompressible fluids; see [46]).

Acknowledgments. The author gratefully thanks Alexander Blokhin for many helpful discussions. The author is indebted to Paolo Secchi for providing him with the reference to [43]. Also, the author thanks Konstantin Ilin and Vladimir Vladimirov for their kind hospitality during his stay at the Hull Institute of Mathematical Sciences and Applications of the University of Hull.

\section{REFERENCES}

[1] M. S. Agranovich, Theorem on matrices depending on parameters and its applications to hyperbolic systems, Funct. Anal. Appl., 6 (1972), pp. 85-93.

[2] S. Alinhac, Existence d'ondes de raréfaction pour des systèmes quasi-linéaires hyperboliques multidimensionnels, Comm. Partial Differential Equations, 14 (1989), pp. 173-230.

[3] A. M. Anile, A. M. Blokhin, and Yu. L. Trakhinin, Investigation of a mathematical model for radiation hydrodynamics, Z. Angew. Math. Phys., 50 (1999), pp. 677-697.

[4] V. B. Baranov, K. V. Krasnobaev, and A. G. Kulikovsky, A model of interaction of the solar wind with the interstellar medium, Soviet Phys. Dokl., 15 (1970), pp. 791-793.

[5] R. Bellman, Introduction to Matrix Analysis, Classics Appl. Math. 19, SIAM, Philadelphia, 1997.

[6] A. M. Blokhin, A mixed problem for a system of equations of acoustics with boundary conditions on a shock wave, Izv. Sibirsk. Otdel. Akad. Nauk SSSR Ser. Tekhn. Nauk, 13 (1979), pp. 25-33 (in Russian).

[7] A. M. Blokhin, Estimation of the energy integral of a mixed problem for gas dynamics equations with boundary conditions on the shock wave, Siberian Math. J., 22 (1981), pp. 501-523.

[8] A. M. Blokhin, A mixed problem for symmetric t-hyperbolic systems of acoustic type, Dinamika Sploshn. Sredy, 52 (1981), pp. 11-29 (in Russian).

[9] A. M. Blokhin, Uniqueness of the classical solution of a mixed problem for equations of gas dynamics with boundary conditions on a shock wave, Siberian Math. J., 23 (1982), pp. 604-615.

[10] A. M. Blokhin, Energy Integrals and Their Applications in Problems in Gas Dynamics, Nauka, Novosibirsk, Russia, 1986 (in Russian).

[11] A. M. Blokhin And V. N Dorovsky, Mathematical Modelling in the Theory of Multivelocity Continuum, Nova Science, New York, 1995.

[12] A. Blokhin and Yu. Trakhinin, Investigation of the well-posedness of the mixed problem on the stability of fast shock waves in magnetohydrodynamics, Matematiche (Catania), 49 (1994), pp. 123-141.

[13] A. Blokhin and Yu. Trakhinin, Stability of strong discontinuities in fluids and MHD, in Handbook of Mathematical Fluid Dynamics, Vol. 1, S. Friedlander and D. Serre, eds., North-Holland, Amsterdam, 2002, pp. 545-652.

[14] A. Blokhin and Yu. Trakhinin, Stability of Strong Discontinuities in Magnetohydrodynamics and Electrohydrodynamics, Nova Science, New York, 2003.

[15] A. M. Blokhin, V. Romano, and Yu. L. Trakhinin, Stability of shock waves in relativistic radiation hydrodynamics, Ann. Inst. H. Poincaré Phys. Théor., 67 (1997), pp. 145-180.

[16] E. Casella, P. Secchi, and P. Trebeschi, Non-homogeneous linear symmetric hyperbolic systems with characteristic boundary, Differential Integral Equations, 19 (2006), pp. $51-74$.

[17] J.-F. Coulombel, Weakly stable multidimensional shocks, Ann. Inst. H. Poincaré Anal. Non Linéaire, 21 (2004), pp. 401-443.

[18] J.-F. Coulombel, Well-posedness of hyperbolic initial boundary value problems, J. Math. Pures Appl., 84 (2005), pp. 786-818.

[19] J.-F. COUlombel AND P. SeCChI, The stability of compressible vortex sheets in two space dimensions, Indiana Univ. Math. J., 53 (2004), pp. 941-1012.

[20] J.-F. Coulombel and P. Secchi, Nonlinear compressible vortex sheets in two space dimensions, Seminario Matematico, Brescia, preprint, 2005. 
[21] J. Francheteau and G. MÉtivier, Existence de chocs faibles pour des systèmes quasilinéaires hyperboliques multidimensionnels, Astérisque, 268, Soc. Math. France, Paris, 2000.

[22] K. O. Friedrichs, Symmetric positive linear differential equations, Comm. Pure Appl. Math., 11 (1958), pp. 333-418.

[23] S. K. Godunov, An interesting class of quasi-linear systems, Soviet Math. Dokl., 2 (1961), pp. 947-948.

[24] S. K. Godunov, Symmetric form of the equations of magnetohydrodynamics, in Numerical Methods for Continuum Mechanics, Vol. 3, Computer Center of the Siberian Branch of the USSR Academy of Sciences, Novosibirsk, 1972, pp. 26-34 (in Russian). English translation at http://citeseer.ist.psu.edu/37221.html.

[25] S. K. Godunov, Equations of Mathematical Physics, Nauka, Moscow, 1979 (in Russian).

[26] T. Kato, The Cauchy problem for quasi-linear symmetric hyperbolic systems, Arch. Ration. Mech. Anal., 58 (1975), pp. 181-205.

[27] H.-O. Kreiss, Initial boundary value problems for hyperbolic systems, Comm. Pure Appl. Math., 23 (1970), pp. 277-296.

[28] L. D. Landau and E. M. Lifshiz, Electrodynamics of Continuous Media. Course of Theoretical Physics, Vol. 8, Pergamon Press, Oxford, 1984.

[29] L. D. Landau and E. M. Lifshiz, Fluid Mechanics. Course of Theoretical Physics, Vol. 6, Pergamon Press, New York and Oxford, 1987.

[30] P. D. Lax, Hyperbolic Systems of Conservation Laws and the Mathematical Theory of Shock Waves, CMBS-NSF Regional Conf. Ser. in Appl. Math. 11, SIAM, Philadelphia, 1973.

[31] D. LI, Rarefaction and shock waves for multi-dimensional hyperbolic conservation laws, Comm. Partial Differential Equations, 16 (1991), pp. 425-450.

[32] A. MAJdA, The Stability of Multi-Dimensional Shock Fronts - A New Problem for Linear Hyperbolic Equations, Mem. Amer. Math. Soc. 41, no. 275, Amer. Math. Soc., Providence, RI, 1983.

[33] A. MaJdA, The Existence of Multi-Dimensional Shock Fronts, Mem. Amer. Math. Soc. 43, no. 281, Amer. Math. Soc., Providence, RI, 1983.

[34] A. Majda, Compressible Fluid Flow and Systems of Conservation Laws in Several Space Variables, Springer-Verlag, New York, 1984.

[35] A. Majda AND S. Osher, Initial-boundary value problems for hyperbolic equations with uniformly characteristic boundary, Comm. Pure Appl. Math., 28 (1975), pp. 607-675.

[36] G. MÉTIVIER, The block structure condition for symmetric hyperbolic systems, Bull. London Math. Soc., 32 (2000), pp. 689-702.

[37] G. MÉTIVIER, Stability of multidimensional shocks, in Advances in the Theory of Shock Waves, Progr. Nonlinear Differential Equations Appl. 47, Birkhäuser, Boston, 2001, pp. 25-103.

[38] G. MÉtivier And K. Zumbrun, Hyperbolic boundary value problems for symmetric systems with variable multiplicities, J. Differential Equations, 211 (2005), pp. 61-134.

[39] J. RAuch, Symmetric positive systems with boundary characteristic of constant multiplicity, Trans. Amer. Math. Soc., 291 (1985), pp. 167-187.

[40] J. B. Rauch AND F. J. Massey, Differentiability of solutions to hyperbolic initial-boundary value problems, Trans. Amer. Math. Soc., 189 (1974), pp. 303-318.

[41] S. SCHOCHET, The compressible Euler equations in a bounded domain: Existence of solutions and the incompressible limit, Comm. Math. Phys., 104 (1986), pp. 49-75.

[42] P. SECCHI, Well-posedness of characteristic symmetric hyperbolic systems, Arch. Ration. Mech. Anal., 134 (1996), pp. 155-197.

[43] P. SECCHI, Inflow-outflow problems for inviscid compressible fluids, Commun. Appl. Anal., 2 (1998), pp. 81-110.

[44] Yu. Trakhinin, A complete 2D stability analysis of fast MHD shocks in an ideal gas, Comm. Math. Phys., 236 (2003), pp. 65-92.

[45] Yu. Trakhinin, On existence of compressible current-vortex sheets: Variable coefficients linear analysis, Arch. Ration. Mech. Anal., 177 (2005), pp. 331-366.

[46] Yu. Trakhinin, On the existence of incompressible current-vortex sheets: Study of a linearized free boundary value problem, Math. Methods Appl. Sci., 28 (2005), pp. 917-945.

[47] A. I. Vol'Pert and S. I. Khudyaev, On the Cauchy problem for composite systems of nonlinear differential equations, Math. USSR-Sb., 16 (1972), pp. 517-544. 